\title{
THE SUMMER MEETING IN LARAMIE
}

The fifty-ninth Summer Meeting of the American Mathematical Society was held at the University of Wyoming, Laramie, Wyoming, from August 31 to September 3, 1954, in conjunction with meetings of the Mathematical Association of America and the Canadian Mathematical Congress.

Over 500 people registered for the meeting including 234 members of the Society.

The Committee to Select Hour Speakers for Annual and Summer Meetings invited five speakers. Tuesday afternoon Professor R. D. James of the University of British Columbia addressed the Society on Integrals and summable trigonometric series. Professor L. V. Ahlfors presided. Professor Charles Loewner of Stanford University addressed the Society Wednesday morning on Some transformations of systems of partial differential equations at a session presided over by Professor D. H. Lehmer. Thursday morning Professor R. S. Phillips of the University of Southern California addressed the Society on Semigroups of operators and Thursday afternoon Professor J. G. Wendel addressed the Society on Semi-groups in algebra at sessions presided over by Professor B. J. Pettis and Professor W. L. Duren respectively. Dr. R. E. Bellman of The Rand Corporation addressed the Society Friday morning on Theory of dynamic programming. Professor C. B. Tompkins presided.

There were fourteen sessions for contributed papers presided over by Professors M. A. Basoco, R. W. Brink, J. W. Hurst, V. L. Klee, C. G. Phipps, G. B. Price, O. F. G. Schilling, Nathan Schwid, M. F. Smiley, Leonard Tornheim, A. W. Tucker, V. J. Varineau, R. M. Winger, and C. R. Wylie.

The Committee on Arrangements provided an enjoyable program for those attending the meeting. Included in these events were: a tea for the ladies on Monday afternoon, movies Monday evening, a trip to Vedauwoo on Tuesday afternoon, a trip to Snowy Range and a wild game steak fry on Wednesday, and a square dance Thursday evening.

On Wednesday evening, at the wild game steak fry, President George Duke Humphrey of the University of Wyoming welcomed the mathematical organizations to Wyoming. Professor W. L. Duren responded for the organizations by presenting resolutions thanking the University of Wyoming and all responsible individuals for their generous and effective contributions to the success of the meeting. 
The Council met on Tuesday evening, August 31, 1954.

The Secretary announced the election of the following ninety-two persons to ordinary membership in the Society:

Mr. David Samuel Adorno, Haller, Raymond and Brown, Inc., State College, Pennsylvania;

Mr. Robert Joseph Arms, New York University;

Mr. Donald Gary Aronson, Massachusetts Institute of Technology;

Mr. Pierre Belanger, 83 Casot Avenue, Quebec, Quebec, Canada;

Mr. Stoughton Bell, University of California, Berkeley;

Mr. John William Blattner, University of California, Los Angeles;

Mr. Mark Clinton Breiter, Ballistic Research Laboratories, Aberdeen Proving Ground, Maryland;

Mr. Delmar Lee Boyer, University of Kansas;

Mr. Robert W. Butcher, University of Michigan;

Mr. Roderick Peter Campbell Caldwell, University of Illinois;

Mr. Benjamin Ralph Cato, Jr., University of Maryland;

Dr. Moody Lee Coffman, Convair Division, General Dynamics Corp., Fort Worth, Texas;

Dr. Louis J. Cote, Columbia University;

Mr. Glen Jacob Culler, University of California, Berkeley;

Mr. Hubert M. Custer, Elizabethtown College;

Mr. Don Allen Davis, University of Washington;

Mr. Joseph DeMilia, U. S. Naval Gun Factory, Washington, D. C.;

Mr. Gus DiAntonio, University of Pittsburgh;

Mr. Russell D. F. Dineen, Board of Public Education, Wilmington, Delaware;

Miss Mary Louise Edwards, Lincoln University;

Professor John Arthur Englund, Creighton University;

Mr. William Paul Evans, Caterpillar Tractor Company, Peoria, Illinois;

Miss Louisa Sonia Grinstein, University of Michigan;

Mr. John Wesley Hamblen, Purdue University;

Miss Florence Ruth Harwich, Columbia University;

Mr. Simon Hellerstein, Syracuse University;

Mr. Robert Bennett Herrera, Los Angeles City College;

Mr. Frederick William Hoagland, 1157 East 57th Street, Chicago, Illinois;

Mr. Harry Hochstadt, W. L. Maxson Corp., New York, New York;

Mr. Henry F. Hunter, Knolls Atomic Power Laboratories, Schenectady, New York;

Mr. Dale Harper Husemoller, Harvard University;

Mr. Amnon Jakimovski, Tel-Aviv, Israel;

Mr. Guy Johnson, Jr., Rice Institute;

Mr. David Hunter Jones, University of Washington;

Mr. John Jones, Jr., Mississippi Southern College;

Mr. Edgar David Kann, New York University;

Lieutenant Jack Herman Kaplan, United States Army, Fort Eustis, Virginia;

Mr. Arthur Alonzo Karwath, Saint Ambrose College, Davenport, Iowa;

Miss Catherine Shannon Kay, North Central College;

Mr. Donald Allen Kearns, University of Maine;

Mr. Maurice Kennedy, California Institute of Technology;

Mr. Richard Frederick King, University of Illinois;

Mr. Walter Charles Koroluk, Olin Industries, New Haven, Connecticut;

Dr. Gregers Louis Krabbe, University of California, Berkeley; 
Mr. Gene Levy, University of Oklahoma;

Professor Chio-Shih Lin, University of the Philippines;

Mr. Richard Gene Long, University of Washington;

Mr. William Caroll Long, Consolidated Vultee Aircraft Corp., Fort Worth, Texas;

Mr. Gerald E. Mahoney, Boston University;

Mr. Mark Edward Mahowald, University of Minnesota;

Dr. Allan Dean Martin, Oberlin College;

Mr. Samuel Melamed, McGill University;

Mr. Roger Edwin Messick, Carnegie Institute of Technology;

Mr. Richard John Mihalek, Illinois Institute of Technology;

Dr. Cordell Bridges Moore, Consolidated Vultee Aircraft Corp., Fort Worth, Texas;

Mr. Hal G. Moore, Carbon Senior High School and Carbon College, Price, Utah;

Professor Mikao Moriya, Okayama University, Okayama, Japan;

Professor David Eugene Muller, University of Illinois;

Mr. Lewis Amedeus Ondis, II, Westinghouse Electric Corp., Pittsburgh, Pennsylvania;

Mother Harriet Ann Padberg, College of the Sacred Heart, Grand Coteau, Louisiana;

Mr. Frank Joseph Palas, Oklahoma University;

Professor Henry Parkus, Michigan State College;

Miss Constance Miller Parson, Fort Valley State College;

Mr. Edward William Payne, William J. Bryan University;

Mr. Arthur Ferris Payton, National Union Radio Corp., Orange, New Jersey;

Professor George W. Peglar, Iowa State College;

Mr. Paul John Persiani, Saint Louis University;

Dr. Lloyd Lewis Philipson, Rand Corporation, Santa Monica, California;

Mr. Bennie H. Pierce, Lees-McRae College;

Mr. José Fortunato Pita de Macedo, Lisbon High School, Lisbon, Portugal;

Dr. Gerald Cowles Preston, Purdue University;

Dr. Royal William Randall, Jr., Purdue University;

Mr. Thomas David Riney, Purdue University;

Dr. Leon C. Robbins, Jr., Burroughs Corp., Philadelphia, Pennsylvania;

Mr. Willy Werner van Roosbroeck, Bell Telephone Laboratories, Murray Hill, New Jersey;

Mr. Paul John Schillo, University of Buffalo;

Mr. Ralph Edgar Schwartz, University of Massachusetts;

Dr. Binyamin Schwarz, Ministry of Defense, Tel-Aviv, Israel;

Mr. Cecil Fay Shelton, Atlantic Refining Company, Caracas, Venezuela;

Dr. Bella Manel Shiffman, Ramo-Wooldridge Corporation, Los Angeles, California;

Mr. Lewis Joseph Simonoff, Syracuse University;

Dr. Frank William Sinden, Aurora, Illinois;

Mrs. Doris Skillman Stockton, University of Massachusetts;

Mr. Malcolm Smith, Cook Research Laboratories, Skokie, Illinois;

Mr. Newton Birrell Smith, 228 Welch, Ames, Iowa;

Mr. Charles Edwart Steward, Boeing Airplane Company, Seattle, Washington;

Mr. Charles Russell Strain, Engineering Research Associates, Inc., Arlington, Virginia;

Professor Lee C. Teng, University of Wichita;

Mr. Louis George Vargo, Convair Division, General Dynamics Corp., Fort Worth, Texas;

Mr. Andries Cometis de Wilde, Detroit Institute of Technology; 
Professor Samuel Watkins Williams, Arkansas College;

Mr. Bevin K. Youse, University of Georgia.

It was reported that the following five persons had been elected to membership on nomination of institutional members as indicated:

University of Florida: Mr. George Van Zwalenberg.

University of Illinois: Mr. James Harman Abbott

Metropolitan Life Insurance Company: Mr. Joseph Thomas Gannon

University of Missouri: Mr. William Robert Abel and Mr. James Fredrik Jakobsen

The Secretary announced that the following had been admitted to the Society in accordance with reciprocity agreements with various mathematical organizations: German Mathematical Society: Professor Guido Hoheisel, University of Köln, Köln, Germany; Indian Mathematical Society: Dr. V. K. Balachandran, Mr. E. de St. Q. Isaacson, and Dr. M. Venkataraman; Polish Mathematical Society: Professor Henry Hiz, Pennsylvania State University; The Swedish Mathematical Society: Professor Harold Bror Evert Bergström, Chalmers University of Technology, Goteborg, Sweden; Wiskundig Genootschap: Dr. Gerard Willem Decnop, University Library, Amsterdam, Holland, and Dr. Herman van Rossum, The Amsterdam Lyceum.

The following appointments by the President were reported: R. V. Churchill appointed Chairman of the Editorial Committee for Applied Mathematics Symposium Proceedings for the period July 1, 1954-June 30, 1955; F. J. Murray appointed a member of the Editorial Committee for Applied Mathematics Symposium Proceedings for a period of three years beginning July 1, 1954 (committee now consists of R. V. Churchill (Chairman), A. E. Heins, and F. J. Murray); J. L. Doob appointed Chairman of the Committee on Translations from Russian and Other Foreign Languages for a period of one year beginning July 1, 1954; R. E. Bellman and Hans Samelson appointed as members of the Committee on Russian and Other Foreign Languages for a period of three years beginning July 1, 1954 (committee now consists of J. L. Doob (Chairman), R. E. Bellman, R. P. Boas, Irving Kaplansky, and Hans Samelson); F. B. Jones appointed Chairman of the Organizing Committee for Summer Institutes; Edwin Hewitt and J. J. Stoker appointed as members of the Organizing Committee for Summer Institutes for a period of three years beginning July 1, 1954 (committee now consists of: F. B. Jones (Chairman), A. A. Albert, Salomon Bochner, S. S. Chern, Edwin Hewitt, and J. J. Stoker); John von Neumann (Chairman), H. W. Bode, Richard Courant, D. H. Lehmer, and T. Y. Thomas appointed as a Program Committee for a Symposium in Applied Mathematics to 
be held on April 14-15, 1955, at Polytechnic Institute of Brooklyn; R. M. Foster (Chairman), L. W. Cohen, S. P. Hoffman, Jr., J. P. Russell and Seymour Schuster appointed as a Committee on Local Arrangements for a meeting to be held at the Polytechnic Institute of Brooklyn, April 14-16, 1955; Professors B. P. Gill and C. H. W. Sedgewick as auditors for 1954.

The following appointments of representatives of the Society were reported: at the inauguration of Francis H. Horn as President of Pratt Institute on May 15, 1954: Professor Samuel Borofsky; at the inauguration of David Hitchens Morgan as President of the Texas Agricultural and Mechanical College on May 20, 1954: Professor E. C. Klipple; at the inauguration of Benjamin Franklin Hilbun as President of Mississippi State College on July 14, 1954: Professor Arthur Ollivier.

The Secretary reported that the following persons had accepted invitations to deliver addresses during 1954: Charles Loewner, Laramie, Wyoming, Summer Meeting 1954; J. G. Wendel, Laramie, Wyoming, Summer Meeting 1954; Arne Beurling, Cambridge, Massachusetts, October 30,1954; Nathan Jacobson, University of Alabama, November 26-27, 1954; Lipman Bers, Pittsburgh, Pennsylvania, Annual Meeting 1954; and Samuel Eilenberg, Pittsburgh, Pennsylvania, Annual Meeting 1954.

The Secretary reported that Professor K. O. Friedrichs had accepted an invitation to deliver the Josiah Willard Gibbs Lecture in December, 1955 and that Professor Salomon Bochner had accepted an invitation to deliver the Colloquium Lectures in 1956.

It was reported to the Council that the Executive Committee and the Trustees had approved a recommendation that Russian Translations be issued in bound volumes of about two hundred to three hundred pages, each containing (whenever feasible) papers on related subjects. The volumes will be issued at irregular intervals, sold separately, and kept in stock, like any other series of books published by the Society. About four volumes a year are contemplated.

The following actions taken by mail vote of the Council were reported: The Riverside Campus of the University of California was elected to institutional membership in the Society. Dr. John H. Curtiss was elected Executive Director of the Society for a term of two years beginning September 1, 1954.

It was reported that the Executive Committee had voted to schedule a joint session of the Society and Section A of the AAAS at the meeting of the AAAS in Berkeley, California, December, 1954.

The final report of the Executive Director Dr. H. M. MacNeille 
was presented. In this report the activities at the Headquarters of the Society during the past five years were reviewed.

The Council voted to set meetings on February 26, 1955 at Columbia University and June 18, 1955 at the University of British Columbia. The Council elected Professors A. M. Gleason and J. L. Kelley as representatives of the Society on the Board of Editors of the Annals of Mathematics for a term of three years beginning January 1, 1955. The Council elected Professors D. H. Lehmer and N. E. Steenrod as representatives of the Society in the Division of Mathematics of the National Research Council for a term of three years beginning July 1, 1955.

The Council voted to extend Professor Saunders MacLane's leave of absence as a member of the Editorial Committee for the Transactions and Memoirs to November 10, 1954.

A Business Meeting of the Society was held in Education Auditorium at 10:15 a.m. September 1, 1954, with Vice President D. H. Lehmer presiding. The Secretary reported on the affairs of the Society.

The members present voted to amend the by-laws as follows:

To amend Article VIII, Section 2 to read: There shall be four classes of members, namely ordinary, contributing, corporate and institutional.

To amend Article VIII, Section 5 to read: A university or college, or a firm, corporation, or association interested in the support of mathematics may be elected a corporate or an institutional member.

To amend Article IX, Section 5 to read: The minimum dues of a corporate member shall be one hundred dollars.

To amend Article IX, Section 6 to read: The privileges of a corporate or an institutional member shall depend on its dues in a manner to be determined by the Council, subject to approval by the Board of Trustees. These privileges shall be in terms of Society publications to be received by the institution and of the number of persons it may nominate for ordinary membership in the Society.

To amend Article IX, Section 11 to read: The annual dues of ordinary, contributing, and corporate members shall be payable after October first of the preceding year and due by January first of the year to which they apply. The Society shall submit bills for dues. If the annual dues of any member remain undischarged beyond what the Board of Trustees deem to be a reasonable time, his name shall, after due notice, be removed from the list of members. If a member wishes to discontinue his membership at any time, he shall submit his resignation in writing to the Society. 
The abstracts of the papers follow. Those having the letter " $t$ " following the abstract number were read by title. Where a paper, presented in person, has more than one author, the symbol (p) follows the name of the author who presented it. Paper number 774 was read by Dr. T. C. Doyle. Professor Jehle and Dr. Bade were introduced by Professor H. B. Ribeiro, Dr. Peaceman and Dr. Rachford by Dr. Jim Douglas, Jr., Mr. Hanf by Professor Alfred Tarski and Dr. Knothe by Professor J. A. Ward.

\section{Algebra ANd Theory of Numbers}

635. T. M. Apostol (p) and Abe Sklar: Identities related to approximate functional equations of certain Dirichlet series.

The authors derive identities expressing any Hecke series $\phi(s)=\sum a(n) n^{-8}$ of signature $(\lambda, \kappa, \gamma)$ in terms of its partial sums and a series involving integrals of Bessel functions. A similar identity is obtained for the product of two Riemann zetafunctions $\zeta(s) \zeta(s+p)$. These identities can be used to obtain approximate functional equations for the Dirichlet series in question. The method employs inverse Mellin transforms and is similar to the technique used by one of the authors [Duke Math. J. vol. 18 (1951) pp. 517-525] to obtain identities of a different type for the same functions. (Received July 19, 1954.)

\section{6t. Rafael Artzy: On loops with a special property.}

Loops $G$ with the property $x y \cdot x^{\prime}=y$ for all $x, y \in G$ are dealt with, $x^{\prime}$ being defined by $x x^{\prime}=1$. As a special device the author uses "cycles," i.e. sequences $x_{1}, \cdots, x_{n}$ such that $x_{i}^{\prime}=x_{i+1} \bmod n$. The main results are the following: (1) $G$ cannot consist only of 1 and of $m$ cycles of equal length $n$, unless $n$ divides $2 m$. Corollary: If $m=1$, right and left inverses coincide. (2) There exist infinitely many nonisomorphic loops $G$ of infinite order whose elements (besides 1) form an infinite cycle. (3) The unit element and the elements of a cycle of length $n$, together with the elements of all the cycles whose lengths are factors of $n$, form a subloop of $G$. (4) If $G$ has a cycle of length $n>2, G$ has another cycle whose length divides $n$. (5) If an infinite $G$ contains finite cycles, then 1 and all elements of the cycles form a subloop of $G$. (6) Finite loops $G$ of identical structure, as regards the number of cycles and their lengths, need not be isomorphic. (Received July 12, 1954.)

637t. H. W. Becker: Duals of the classic rational cuboids, and Hero and Pythagorean triangles with two sides in common.

R. D. Carmichael, Diophantine analysis, p. 103, prob. 9, made the even power case of Fermat's Last Theorem depend on (1) $x^{2}+y^{2}=z_{1}^{2}, x_{1}^{2}+y^{2}=z^{2}, z^{2}+y_{1}^{2}=z^{2}$, a tetrahedron with $3 \mathrm{rt}$. $\angle \mathrm{s}$ each at a different vertex. Dualize the Saunderson cuboid, M. Kraitchik, Scripta Math. vol. 11 (1945) p. 322, by reversing alternate signs, then apply $r, s \rightarrow r \pm s$. (1) is thus satisfied by (2): $x, z=\left(r^{2} \mp s^{2}\right)\left(r^{4} \pm r^{2} s^{2}+s^{4}\right) ; y=2 r s\left(r^{4}-s^{4}\right)$; $x_{1}, z_{1}=\left(r^{2} \pm s^{2}\right)\left(r^{4} \mp 3 r^{2} s^{2}+s^{4}\right) ; y_{1}=2 r^{3} s^{3} ;=63,65,60,25,87,16$ in the smallest example. The tet. is physically unrealizable unless $x_{1}+y_{1}>z_{1}$, met by $r, s=4$, 3 , etc.; each side of the inequality is divisible by $r-s$. FLT further requires $x_{1}, y_{1}, z_{1}=p$ th powers, $p>1$ : it is conjectured that at most 2 of the 6 values can be such. The Euler transforms of (1), (2) are the tets. with $2 \mathrm{rt}$. $\measuredangle$ at one vertex symmetrical to the $3 \mathrm{rd}$ 
rt. $\angle$ (3) $X^{2}+Y^{2}=Z_{1}^{2}, Z^{2}=Y^{2}-X_{1}^{2}=X^{2}-Y_{1}^{2}$, and (4) $X, Z=2 r s\left(r^{2} \pm s^{2}\right)\left(r^{4} \mp r^{2} s^{2}+s^{4}\right)$; $Y=\prod\left(r^{4} \pm r^{2} s^{2}+s^{4}\right) ; \quad X_{1}, Z_{1}=\left(r^{4} \pm r^{2} s^{2}+s^{4}\right)\left(r^{4} \mp 3 r^{2} s^{2}+s^{4}\right) ; \quad Y_{1}=4 r^{4} s^{4} ; \quad X_{1}+Y_{1}$ $=\left(r^{4}-r^{2} s^{2}+s^{4}\right)^{2} ;=260,252,273,105,377,64,169$ in the least example. In the least real tet. $r, s=5,4$. Removing $Z_{1}$ and dividing out $r^{4}-r^{2} s^{2}+s^{4}$, the tet. flattens down to a Hero $\triangle h_{1}, b, h_{2}$ of alt. $=a$ and base-segments $=b_{1}, b_{2}$, having 2 sides in common with a Pyth. $\triangle h_{1}, b, h$. With $b=b_{1}+b_{2}, h=\left(h_{1}^{2}+b^{2}\right)^{1 / 2}$, and $H, P$ the respective areas, one gets (5), $a, h_{1}=2 r s\left(r^{2} \mp s^{2}\right) ; b, h_{2}=r^{4} \pm r^{2} s^{2}+s^{4} ; b_{2}, h=r^{4} \mp 3 r^{2} s^{2}+s^{4} ; b_{1}=4 r^{2} s^{2}$; $H, P=r s\left(r^{2} \mp s^{2}\right)\left(r^{4}+r^{2} s^{2}+s^{4}\right)$, generalizing the Hero and Pythagorean triangle pair 20, 21, 13 and 20, 21, 29. (Received May 20, 1954.)

\section{8t. H. W. Becker: Hero and Diophantus quadrilaterals with edges} squares.

Their diagonals are respectively $2(i j k l)^{1 / 2}=$ int., and $\left\{\left(i^{2}+j^{2}\right)\left(k^{2}+l^{2}\right)\right\}^{1 / 2}$; they have the same 4 integer edges $i k \pm j l, i l \mp j k$. All 4 are $\$$ in the Euler (5) and Gerardin solutions of $A^{4}+B^{4}=C^{4}+D^{4}$, Dickson's History II, pp. 646-647; $i, j, k, l$ are resp. $f^{8}+20 f^{6} g^{2}+86 f^{4} g^{4}+20 f^{2} g^{6}+g^{8}, \quad 4 f g\left(f^{2}+g^{2}\right)\left(f^{4}-18 f^{2} g^{2}+g^{4}\right), \quad 4 f^{6}+f^{4} g^{2}+10 f^{2} g^{4}+g^{6}, f^{6}$ $+10 f^{4} g^{2}+f^{2} g^{4}+4 f^{6}, \quad$ and $\left(f^{2}+g^{2}\right)\left(f^{4}+3 f^{2} g^{2}+g^{4}\right), \quad 6 f^{3} g^{3}, \quad f^{2}\left(f^{6}-2 f^{4} g^{2}+f^{2} g^{4}+g^{6}\right)$, $g^{2}\left(f^{6}+f^{4} g^{2}-2 f^{2} g^{4}+g^{6}\right)$. These are the same quads. under $f, g \rightarrow f \pm g$; but are based on distinct solutions of $p q\left(p^{2}+q^{2}\right)=r s\left(r^{2}+s^{2}\right)$. " $2379^{4}+27^{4}=729^{4}+577^{4}$ " is an erratum; the intended meaning was the quad. of Desboves ${ }^{169}$ and its self-transform $A \pm D$, $C \pm B=2379,27,729,577=p^{\prime}, q^{\prime}, r^{\prime}, s^{\prime}$. In Fauquembergue's ${ }^{178}$ solution of $A^{2}+B^{4}$ $=C^{4}+D^{4}$, ibid., the last 3 of $i k \mp j l, i l \pm j k$ are [s, with $i, j, k, l=f^{2}+16 g^{2}, f^{2}+12 f g$ $+4 g^{2}, 6 f(f-g), 10 f^{2}+6 f g+g^{2}$. To make $i k-j l, i l-j k$ [s], put $i, k, j, l=f(f \pm g)$, $g(f \mp g)$. Or, let $i, j, k, l=I^{2}, J^{2}, K^{2}, L^{2} ; I, J, K, L$ parameters of a quad. with 4 edges \$. Or, satisfy $a b\left(c^{2}-d^{2}\right), c d\left(a^{2}-b^{2}\right)=\square$, met by parameters of any Petrus vector or its double; perhaps the simplest solution of this type is the Euler-Hillyer $i, j, k, l$ $=\left(3 r^{2}+s^{2}\right)\left(3 r^{2}-s^{2}\right)^{2}(3 r \pm s)(r \pm s), 16 r^{2} s^{2}(3 r \mp s)(r \mp s)$. From one numerical solution of $A^{2}+B^{2}=C^{4}+D^{4}$, hence $i k+j l, i l-j k=\$$, another is derived, etc. ad inf., by Carmichael's transform, Diophantine analysis, p. 47. Thus $39^{2}+20^{2}=6^{4}+5^{4} \rightarrow 42961^{2}$ $+54080^{2}=119^{4}+260^{4}$. Parametric solution is a desideratum. So are $D Q$ with 2 or more edges $m$ th powers. (Received May 20, 1954.)

639t. H. W. Becker: Transforms from automedian triangles with two sides squares to Pythagorean tetrahedrons.

(1) $2 T^{4}-S^{4}=R^{2}$, an $A \triangle$ with 2 sides [S]; recurrence solutions, Dickson's History II, pp. $620-627$ (errata: p. 622 last line, $\xi, \eta$ should be $y, x ;$ p. 625 last line, $\sigma^{2} x^{2}$ should be $\sigma^{2} x$; p. 626 line $34,8 \lambda h k$ should be $8 \lambda \mu h k$ ). (2) $v^{2}+x^{2}=u^{2}+z^{2}=t^{2}, v^{2}-z^{2}=u^{2}-x^{2}$ $=y^{2}$, a PT. Landen, ibid. p. 447 (erratum: "The case $f=2$ gives Bumpkin's answer" isn't true till 2 sentences later) last method, is generalized to a transform (1) $\rightarrow$ (2) $x=T R S^{3}, z=\left(R^{2}-S^{4}\right) T^{2} / 2 ; y, u=\left(R^{2} \mp S^{2} T^{2}\right) S^{2} / 2 ; v, t=\left(T^{2} R^{2} \mp S^{6}\right) / 2$. Thus $T, S, R$ $=13,1,239 \rightarrow x, z, y, u, v, t=3107,4826640,28476,28645,4826724,4826725$; this specimen is unique, in that $t$ and $v$ (and most of the important parameter pairs) differ by unity. Frenicle's problem ibid. pp. 184-186: (3) $X^{2}+Y^{2}=T^{2}-Z^{2}=U^{2}$, $W=X \pm Y=Z \pm U$ (in some combo), $S=X \mp Y, V=Z \mp U$, depends on (1). A solution of (4) $a b c d\left(a^{2}-b^{2}\right)\left(c^{2}-d^{2}\right)=\square=y^{2} / 16$ is $a, b, c, d, a \pm b, c \pm d=Z, U, U V, Z W, W, V$, $T^{2}, S^{2}$. E.g. if $T=S=R=1$, then $U, X, Y, Z, T=5,3,4,12,13$ and $a, b, c, d=12,5,85$, 84, parameters of a PT. This triadizes with the Petrus transform of the Landen PT, whose $a, b, c, d, a \pm b, c \pm d=U V, Z W, T^{4}, 4 Z U V W, T^{2}, S^{2}, R^{2}, S^{4}$. (3) is a Petrus transform (5) $T, U=t^{2} \pm y^{2} ; U, X=x^{2} \pm z^{2} ; Y=2 x z, Z=2 t y$ of a $3 D$ Diophantine vec- 
tor (6) $x^{2}+y^{2}+z^{2}=t^{2}$, such that $z(x \pm z)=t y, x^{2} \pm 4 t y=w^{2}$. With $\left(1^{\prime}\right) S^{\prime 4}-2 T^{\prime 4}=R^{\prime 2}$, and subscript 0 denoting the next smaller solution of (1), (3), then (7) $T_{0}^{\prime} / S_{0}^{\prime}=\left(T_{0} S_{0}\right.$ $\left.\pm R_{0}\right) /\left(2 T_{0}^{2}+S_{0}^{2}\right) ; w, x, y, z, t=T_{0} T_{0}^{\prime} \pm S_{0} S_{0}^{\prime}, S_{0} T_{0}^{\prime}, T_{0} T_{0}^{\prime}, T_{0} S_{0}^{\prime}$. Fermat's $P \triangle$ is (8) $P^{2}+Q^{2}=T^{4}, P \pm Q=S^{2} ; P, Q=\left(S^{2} \pm R\right) / 2=2 Z U, V W$ in some order. The $A$ s needn't be realizable. (Received July 1, 1954.)

\section{0t. Garrett Birkhoff and R. S. Pierce: Lattice ordered rings.}

A po-ring is an (associative) ring which is partly ordered such that $x \geqq y$ implies $a+x \geqq a+y$ for all $a$, and $a \geqq 0, b \geqq 0$ implies $a b \geqq 0$. An 1-ring is a po-ring which is a lattice under its partial ordering. An f-ring is an 1-ring which satisfies: $b \wedge c=0$ implies $a b \wedge c=b a \wedge c=0$ for all $a \geqq 0$. Lemma: An l-ring is an f-ring iff $(a \vee 0) \wedge(-a(b \bigvee 0) \vee 0)$ $=0$ and symmetrically for all $a$ and $b$. Corollary: The class of f-rings is closed under the formation of subalgebra, direct union and homomorphic image. Theorem: Any 1-ring [ $\sigma$-complete 1-ring] with a unity which is a strong [weak] order unit is an f-ring. Theorem: An f-ring is subdirectly indecomposable iff it is a (simply) ordered ring. Corollary: Any f-ring is isomorphic to a subdirect union of ordered rings and, conversely, any subdirect union of ordered rings is an f-ring. (Received April 5, 1954.)

641. R. H. Bruck and L. J. Paige (p): Loops whose inner mappings are automorphisms.

Let $G$ be a loop and let $I^{*}$ be the inner mapping group of $G$. If every element of $I^{*}$ is an automorphism of $G, G$ is said to be an $A$-loop. $A$-loops have the following properties: (i) Subloops and quotient loops are $A$-loops; (ii) Characteristic subloops are normal; (iii) Every maximal associative (commutative) subset is a subloop; (iv) $A$-loops are power associative. A necessary and sufficient condition that every isotope of an $A$-loop is again an $A$-loop is that $N \supseteq G^{\prime}$, where $N$ is the nucleus and $G^{\prime}$ the derived loop of $G$. The similarity between diassociative $A$-loops and Moufang loops is shown. (Received July 14, 1954.)

642t. Leonard Carlitz: Weighted quadratic partitions over $G F[q, x]$.

For $\alpha \in G F(q), q=p^{n}, p>2$, define $e(\alpha)=e^{2 \pi i t / p}$, where $t=\alpha+\alpha^{p}+\cdots+\alpha^{p^{n-1}}$. For $\xi=\sum_{-\infty}^{m} \alpha_{i} x^{i}, \alpha_{i} \in G F(q)$, define $e(\xi)=e\left(\alpha_{-1}\right)$. Now construct the sum $S=\sum e\left(\xi_{1} U_{1}\right.$ $\left.+\cdots+\xi_{r} U_{r}\right)$, where the summation is over all $U_{i} \in G F[q, x]$ of degree $<k$ such that $\alpha_{1} U_{1}^{2}+\cdots+\alpha_{r} U_{r}^{2}=M$. In the present paper it is shown that $S$ can be evaluated in terms of Gauss and Kloosterman sums. For the special case of weighted quadratic partitions over a finite field see Canadian Journal of Mathematics vol. 5 (1953) pp. 317-323. (Received July 12, 1954.)

\section{3t. Leonard Carlitz: Some applications of weighted quadratic par- titions. \\ Making use of the explicit results for weighted quadratic partitions in a finite field (Canadian Journal of Mathematics vol. 5 (1953) pp. 317-323) it is shown for example how to determine the number of solutions $\xi_{1}, \cdots, \xi_{r}$ of the equation $\alpha=\alpha_{1}\left(\xi_{1}^{p}-\xi_{1}\right)^{2}+\cdots+\alpha_{r}\left(\xi_{r}^{p}-\xi_{r}\right)^{2}$, where $\alpha, \alpha_{i}, \xi_{i} \in G F(q)$. (Received July 12, 1954.)}

644t. Chen-Chung Chang: Two theorems on direct decompositions of relations. Preliminary report.

A relation $R$ is said to be connected if $R \neq 0$ and whenever $R=S \cup T$ where the fields of $S$ and $T$ are disjoint, then either $S=0$ or $T=0$. The following theorems are proved: (I) If $R$ is a connected reflexive relation which is anti-symmetric, then the 
set of factor congruence relations over $R$ forms a Boolean algebra under relative multiplication and set intersection. (See B. Jonsson and A. Tarski, Bull. Amer. Math. Soc. Abstract 59-1-114.) (II) If $R$ is a connected reflexive relation which has at least one anti-symmetric element $x$, i.e., such that the formulas $x R y$ and $y R x$ imply $x=y$, then $R$ has the refinement property, that is to say, if $R \cong \mathfrak{B}_{i} \in I{ }_{I} S_{i} \cong \mathfrak{P}_{j} \in{ }_{J} T_{j}$, then there are relations $U_{\langle i, j\rangle},\langle i, j\rangle \in I \times J$, such that $S_{i} \cong \mathfrak{P}_{j \in J} U_{\langle i, j\rangle}$ for every $i \in I$ and $T_{j}$ $\cong \mathfrak{B}_{i \in I} U_{\langle i, j\rangle}$ for every $j \in J$. It is known that for every relation $R$ the conclusion of (I) implies the conclusion of (II); also the conclusion of (I) implies $R$ has at most one decomposition into indecomposable factors while the conclusion of (II) implies $R$ has up to isomorphism at most one such decomposition. Theorems (I) and (II) improve various known factorization theorems for relations (J. Hashimoto, Ann. of Math. vol. 54 (1951) pp. 315-318; J6nsson and Tarski, op. cit.; Tarski, Cardinal algebras, p. 309). Similar results for algebras $\mathfrak{A}=\langle A,+\rangle$ have also been obtained. (Received July 19, 1954.)

\section{5t. Harvey Cohn: Finiteness of representations by binary abelian} forms. Preliminary report.

As an illustration let the form in $(x, y)$ be $N(\omega)$ where $\omega=x-\theta y$ for $\theta$ an integer in an abelian field contained in $K_{f}=R(\exp 2 \pi i / f)$ for $f$ a prime. Then $\omega$ lies in $K_{f}$ and is in constant ratio to another integer $\Omega$ of $K_{f}$ with zero trace. Hence any bound on $N(\omega)$ would yield from $\Omega$ normal bases of submodules of integers with bounded index. Such $\Omega$ are then bounded in size and number, by normal bases. (Received July 19, 1954.)

\section{W. R. Cowell: Quasinormal kernels of loops.}

Let $G$ be a loop and $K$ be the kernel of a homomorphism of $G$ upon a groupoid. Then $K$ is said to be quasinormal in $G$ if the cosets of the homomorphism have the form $x \Delta(K)$, where $\Delta(K)$ is the semigroup of mappings of $G$ generated by $x \rightarrow k x$, $x \rightarrow x k$, where $x \in G, k \in K$. If $C(x)$ is the coset containing $x$ in any homomorphism of $G$ and $K$ is quasinormal, then $C(x) \Delta(K)$ is the coset of a homomorphism of $G$ and $C(x) \Delta(K)=C(x \Delta(K))$. The product of two quasinormal kernels $H$ and $K$ is defined to be $P(H, K)=H \Delta(K)=K \Delta(H)$ and it is shown that $P(H, K)$ is quasinormal in $G$. If $H \cap K=1$, then $P(H, K)=H \times K$. For any two series of quasinormal kernels of $G$, there exist kernels (not necessarily quasinormal) of the subloops in the series which may be intercalated to produce isomorphic refinements. If $M$ is a groupoid possessing an identity and having the property that if $a, b \in M$ the equations $a x=b, y a=b$ have solutions (not necessarily unique) $x, y \in M$, then $M$ is the homomorphic image of at least one loop such that the kernel of the homomorphism is quasinormal. (Received July 19, 1954.)

\section{R. B. Crouch: Monomial groups.}

Let $U$ be a set. Let $H$ be a group. The set of monomial substitutions on the elements of $U$ over $H$ form a group $\Sigma$. The set $V$ of pure multiplications forms a normal subgroup, the basis group. The set $S$ of pure permutations forms a subgroup, the permutation group. $\Sigma$ splits over $V ; \Sigma=V \cup S, V \cap S=E$, where $E$ is the identity of $\Sigma$. Ore [Trans. Amer. Math. Soc. vol. 5, pp. 15-64] has found all representative groups of the splitting over $V$, all normal subgroups of $\Sigma$, and all automorphisms of $\Sigma$ when $U$ is finite. This paper extends his investigation by allowing $U$ to be of arbitrary order. All representative groups of the splitting over $V$ are found for $\Sigma$ and some of its subgroups, including the case when $S$ is replaced by $A$, the infinite alternating group. With a rather strong restriction on elements of $V$ and $S$ or $A$ all normal subgroups 
are determined. All representative groups of the splitting over $V_{n}$ for $\Sigma_{n, A}=V_{n} \cup_{A_{n}}$, where $A_{n}$ is the finite alternating group, are found. The normal subgroups of $\Sigma_{n, A}$, $n \geqq 5$, are determined. That the basis group is characteristic is proved for the cases where the normal subgroups are known. (Received June 17, 1954.)

\section{8t. D. O. Ellis: IPIC representation of lattice automorphisms.}

It may be concluded from previous studies (Bull. Amer. Math. Soc. Abstracts $60-1-89,60-1-88)$ that the IPIC mappings of a topolattice form a group under "dual convolution" and that this group is isomorphic to the automorphism group of the topolattice. In the present paper this result is extended to arbitrary bounded lattices (the boundedness being no restriction from the automorphism viewpoint). One may remark that the Boolean algebra of subsets of an infinite set has the same automorphism group as a certain nonmodular lattice and, hence, that automorphisms determine the algebraic character of a lattice very little. (Received June 16, 1954.)

649. Paul Erdös, Leonard Gillman and Melvin Henriksen (p): An isomorphism theorem for real-closed fields.

It is well known that every real-closed field has a unique ordering (which is preserved under isomorphism). An ordered set $L$ is called an $\eta_{\alpha^{-}}$set if (i) no $A C L$ of power $<\aleph_{\alpha}$ is cofinal or coinitial with $L$, and (ii) if $A, B \subset L$, with $|A|,|B|<\aleph_{\alpha}$ and $A<B$, then there is an $x \in L$ such that $A<x<B$. All $\eta_{\alpha}$-sets of power $\aleph_{\alpha}$ are similar (Hausdorff, Grundzïge der Mengenlehre, pp. 180-182). Theorem 1: All real-closed fields that are $\eta_{\alpha}$-sets of power $\boldsymbol{\aleph}_{\alpha}$, where $\alpha>0$, are isomorphic. Next, let $M$ be any maximal ideal of the ring $C(X)$ of all continuous real-valued functions on a completely regular space $X$. Then the residue class field $C(X) / M$ is a real-closed field containing the real field $R$; if $C(X) / M$ contains $R$ properly, then $M$ and $C(X) / M$ are called hyper-real (Hewitt, Trans. Amer. Math. Soc. vol. 64 (1948) pp. 45-99; Isbell, Proc. Amer. Math. Soc. vol. 5 (1954) (to appear)). Theorem 1 is used in obtaining Theorem 2: If $\mathbf{c}=\boldsymbol{N}_{1}$, then all hyper-real fields of power $c$ are isomorphic. Corollary: If $c=\aleph_{1}$, then all the nonreal residue class fields of maximal ideals of a countable complete direct sum of real fields are isomorphic. (Received July 19, 1954.)

650t. Paul Erdös, Leonard Gillman and Melvin Henriksen: Hyperreal fields of various cardinals.

Terminology is as in preceding abstract. Several results in general set theory, concerning decompositions of sets, are derived, and are applied to obtain the following theorems. Theorem 1: Let $X$ be any infinite discrete space; then there exist hyper-real ideals $M$ of $C(X)$ for which $|C(X) / M|=c$. (If $\mathrm{c}=\boldsymbol{N}_{1}$, then by Theorem 2 of the preceding abstract, all such fields $C(X) / M$ are isomorphic.) Theorem 2: Let $X$ denote the discrete space of power $\mathrm{c}$, and let $M$ be any maximal ideal of $C(X)$ such that for every $f \in M$, the subset of $X$ on which $f$ vanishes is of power $\mathfrak{c}$; then $\mathfrak{c}<|C(X) / M| \leqq 2^{c}$. Theorem 3: Let $\mathfrak{m}, \mathfrak{n}$ satisfy $\mathfrak{c} \leqq \mathfrak{n} \leqq \mathfrak{m}$, and let $X$ denote the discrete space of power $\mathfrak{m}$; then there is a maximal ideal $M$ of $C(X)$ for which $\mathfrak{n}<|C(X) / M| \leqq 2^{\mathfrak{n}}$. Corollary: If $X$ is the discrete space of power $\mathfrak{m} \geqq \mathfrak{c}$, then not all hyper-real residue class fields $C(X) / M$ are isomorphic. (Received July 19, 1954.)

651t. William Hanf: A result concerning isomorphism of Boolean algebras. Preliminary report.

The following result (solving a problem of $\mathrm{A}$. Tarski) has been established: (I) $\mathfrak{B}_{1}$, 
$\mathfrak{B}_{2}$ being two-element Boolean algebras, there exists a Boolean algebra $\mathfrak{A}$ such that (i) $\mathfrak{A}$ is isomorphic to the direct product $\mathfrak{A} \times \mathfrak{B}_{1} \times \mathfrak{B}_{2}$ and (ii) $\mathfrak{A}$ is not isomorphic to $\mathfrak{A} \times \mathfrak{B}_{1}$. In fact, $\mathfrak{A}$ is the set-algebra consisting of all those sets of integers which are unions of an arbitrary finite set and of arbitrarily many pairs $\{2 n, 2 n+1\}$. In topological translation (I) yields: (I') There exists a 0 -dimensional compact space $S$, with infinitely many isolated points, such that ( $\left.\mathrm{i}^{\prime}\right) S$ is homeomorphic to each of its subspaces obtained by removing two isolated points, and (ii') $S$ is not homeomorphic to any subspace obtained by removing one isolated point. (I) is related to an earlier result of Shin-ichi Kinoshita who constructed three infinite, denumerable Boolean algebras $\mathfrak{A}, \mathfrak{B}_{1}, \mathfrak{B}_{2}$ satisfying (i), (ii). R. L. Vaught observed that no compact separable space, with infinitely many isolated points, satisfies (ii'); hence $\mathfrak{B}_{1}, \mathfrak{B}_{2}$ being two-element Boolean algebras, no denumerable Boolean algebra $\mathfrak{A}$ satisfies both (i) and (ii). Using a different method, B. Jonsson recently constructed a compact nonseparable space, with infinitely many isolated points, satisfying (ii'); his space however is not 0 -dimensional. (Received July 23,1954 .)

\section{2t. W. E. Jenner: On integral subrings of Lie algebras.}

It is shown that a maximal integral subring of a semisimple Lie algebra over the quotient field of a Dedekind ring of characteristic zero is not necessarily a multiplicative unit operator on its ideals. For terminology see Bull. Amer. Math. Soc. Abstract 60-2-194. Let $k$ be the rational numbers, $D$ the rational integers, and $\&$ the Lie algebra of $n \times n$ matrices of trace zero with $n \geqq 4$. Let $\mathfrak{D}$ be the integral subring of $\mathbb{R}$ spanned over $\mathfrak{O}$ by the elements $e_{i j}(i \neq j)$ and $e_{i i}-e_{n n}(i \neq n)$ where the $e_{\mathrm{rs}}$ are the canonical basis elements for $[k]_{n}$. Let $\alpha=\sum_{i=1}^{n-1}\left(e_{i i}-e_{n n}\right)$ and set $\mathfrak{A}=n \cdot \mathfrak{D}+\mathfrak{D}\{\alpha\}$. Then $[\mathfrak{O}, \mathfrak{A}] \subset \mathfrak{A}$. If $\beta=n^{-1} \alpha$ then $\mathfrak{D}_{m}=\mathfrak{D}+\mathfrak{O}\{\beta\}$ is the unique maximal integral subring containing $\mathfrak{D}$ and $\left[\mathfrak{D}_{m}, \mathfrak{D}_{m}\right] \subset \mathfrak{D}_{m}$. Thus neither $\mathfrak{D}$ nor $\mathfrak{D}_{m}$ is a multiplicative unit operator on its ideals. (Received July 14, 1954.)

653t. Bjarni Jónsson: There exist nonrepresentable integral relation algebras.

(Received July 15, 1954.)

654t. Irving Kaplansky: The Zariski topology and algebraic matrix groups.

Assume: (1) a $T_{1}$-space with descending chain condition on closed sets, (2) a group with continuous inverse and multiplication separately continuous, (3) for fixed $a$, the mapping $x \rightarrow x^{-1} a x$ continuous. Matrix groups under the Zariski topology satisfy these axioms, and a portion of their theory is obtainable this way. Among the byproducts there are several apparently new results such as: in any matrix group there is an upper bound to the size of the finite conjugate classes. (Received July 19,1954.)

655t. Joachim Lambek and Leo Moser: Some associative operations on integers.

Let $f(0)=0, f(1), f(2), \cdots$ be an increasing sequence of non-negative integers, such that $\Delta f(a)+\Delta f(b) \leqq \Delta f(a+b)+1$, where $\Delta f(m)=f(m+1)-f(m)$. Let $g(n)$ be the largest $m$ for which $f(m) \leqq n$. Then the operation $m \circ n=m+n+f(g(m)+g(n))$ $-f(g(m))-f(g(n))$ is associative. This is shown by considering the (1-1) mapping $n \rightarrow(g(n), n-f(g(n))$ of the non-negative integers into the first quadrant of the Cartesian plane, under which $m \circ n$ corresponds to the vector sum of the images of 
$m$ and $n$. An example is $m \circ n=m+n+2\left\{m^{1 / 2}\right\}\left\{n^{1 / 2}\right\}$, where $\{x\}$ is the closest integer to $x$. (Received July 6,1954.)

\section{W. J. LeVeque: The error term in the distribution (mod 1) of $\left\{2^{k} t\right\}$.}

For $0<x<1$ and $0<t<1$, let $N_{n}(x, t)$ be the number of positive integers $k \leqq n$ such that $\left(2^{k} t\right) \leqq x$, where $(u)=u-[u]$. It is well known that the sequence $\left\{\left(2^{k} t\right)\right\}$ is uniformly distributed $(\bmod 1)$, so that $\lim _{n \rightarrow \infty} N_{n}(x, t) / n=x$, for almost all $t$. Put $y_{n}(x, t)$ $=n^{1 / 2}\left(N_{n}(x, t) / n-x\right)$. It is shown that if $t$ is regarded as a random variable with uniform distribution in $(0,1)$, then $\lim _{n \rightarrow \infty} \operatorname{Pr}\left\{\int_{0}^{1} y_{n}^{2}(x, t) d x<\alpha\right\}$ exists and equals $\operatorname{Pr}\left\{\int_{0}^{1} y^{2}(x, t) d x<\alpha\right\}, y(x, t)$ a Gaussian stochastic process with a rather complicated covariance function. A similar result is obtained for $\lim _{n \rightarrow \infty} \operatorname{Pr}\left\{\int_{0}^{1} \phi_{\beta}\left(y_{n}(x, t)\right) d x\right.$ $<\alpha\}$, where $\phi_{\beta}(u)$ is 1 or 0 according as $u \geqq \beta$ or $u<\beta$. Some support is given for the conjecture that the classical discrepancy $\sup _{0<x<1}\left|y_{n}(x, t)\right|$ also has a nondegenerate limiting distribution. (Received July 19, 1954.)

\section{Gene Levy: Representation theory for generalized p-rings.}

A ring $R$ is called a $q$-ring if (1) $x \in R$ implies $x^{q}=x$, and (2) $t<q$ implies there is a $y \in R$ such that $y^{t} \neq y$. The characteristic is finite. Using a theorem of Birkhoff's, it is shown that every $q$-ring is isomorphic to a subdirect sum of Galois fields; the converse is true if the set of distinct Galois fields is finite. For a given $q$ there may be no $q$-ring; the necessary and sufficient condition for the existence of a $q$-ring is that there be primes $p_{i}$ and positive integers $\alpha_{i j}$ such that $q=1+$ l.c.m. ${ }_{i=1,2}, \cdots, n ; j=1,2, \cdots, m\left(p_{i}^{\alpha_{i j}}-1\right)$. A $q$-ring of characteristic $c$ is called a $(q, c)$-ring. For these rings the $p_{i}$ are the prime factors of $c$. Consider the set $S$ of all divisors of $(q-1)$ of the form $\left(p_{i}^{\alpha i}-1\right), p_{i} \mid c$. If $\left(p_{i}^{\alpha_{i}}-1\right) \in S$, there is a $(q, c)$-ring whose representation includes $G F_{p_{i}}^{\alpha_{i}}$. Every representation of a $(q, c)$-ring will include $G F_{p_{i}}$ if and only if $\left(p_{i}-1\right) \in S,\left(p_{i}^{\alpha_{i}}-1\right) \notin S$, $\alpha_{i}>1$; it will include $G F_{p_{i}}^{\alpha_{i}}$ if and only if 1.c.m. $\left\{S-\left(p_{i}^{\alpha_{i}}-1\right)\right\}<(q-1)$. (Received May 18, 1954.)

658. R. J. Mihalek (p) and L. R. Wilcox: The Jordan chain theorem for certain nonmodular lattices.

It is known that the Jordan theorem, to the effect that principal chains with the same end points have equal lengths, holds in any modular lattice, also in any lattice called semi-modular by Birkhoff (Lattice theory, rev. ed., pp. 100, 66-67). This same theorem is now proved for any lattice in which modularity is symmetric, whether of finite length or not. Various consequences, already proved for such lattices of finite length, notably covering relations involving special elements such as points (elements covering 0 ), lines (elements covering points), and hyperplanes (elements covered by 1) are shown to hold in this somewhat more general environment. (Received July 9, 1954.)

\section{9t. Leo Moser and Max Wyman: On an array of Aitkin.}

Let $A_{m, n}$ be defined by (i) $A_{m, n}=A_{m-1, n+1}+A_{m-1, n}(n \geqq 1)$, (ii) $A_{0, n+1}=A_{n, 0}$, (iii) $A_{0,0}=a, A_{0,1}=b$. The case $a=b=1$ has been considered by A. C. Aitkin, $A$ problem in combinations, Math. Notes Edin. vol. 21 (1933) pp. 18-23, and others. Generally, if $A_{0, n}=B_{n}$ then $A_{m, n}=(B+1)^{m} B^{n}$, symbolically, and we need only study the $B$ 's. The general case is reduced to the case $a=0, b=1$. In this case the following results are obtained: (1) $B_{n+1}=(B+1)^{n}-1$. (2) $\sum_{n=0}^{\infty} B_{n} x^{n} / n !=e^{e^{x}} \int_{0}^{x} e^{-e} t d$. (3) $B_{n}$ 
$=\sum_{r=1}^{n} \Delta^{r} 0^{n}\left(0 !-1 !+2 !-\cdots+(-1)^{r-1}(r-1) !\right)$. (4) For $p$ a prime $B_{p} \equiv-(1 / 1$ ! $+1 / 2 !+\cdots+1 /(p-1) !)(\bmod p)$. (Received July $19,1954$.

\section{0t. Leo Moser and Max Wyman: On solutions of $x^{d}=1$ in sym- metric groups.}

Let $A_{n, d}$ denote the number of solutions of $x^{d}=1$ in the symmetric group of degree $n$. A relation is established between $A_{n, 2}$ and the Hermite polynomials $H_{n}(x)$. This leads to a refinenent of some results of Chowla, Herstein, and Moore (Canadian Journal of Mathematics vol. 3 (1951) pp. 328-334). By another method, asymptotic formulae for $A_{n, p}$ are obtained. Some arithmetic properties of $A_{n, d}$ are proved, extending results of the paper mentioned above and of results proved by E. Jacobsthal (Norske Vid. Selsk. vol. 21 (1949) pp. 49-51) and Chowla, Herstein, and Scott (Norske Vid. Selsk. vol. 21 (1952) pp. 49-51). It is shown how some of these results can be extended to alternating groups. (Received July 19, 1954.)

\section{H. B. Ribeiro: Boolean algebras with operators and topological} groups. Preliminary report.

Complex algebras of groups, as $T_{1}$ spaces, have been discussed as, respectively, special relation algebras and special closure algebras. (For this and terminology see ChinTarski, Distributive and modular laws in the arithmetic of relation algebras, University of California Publications in Mathematics, 1951, and Jonsson-Tarski, Boolean algebras with operators, I and II, Amer. J. Math. vols. 73 and 74.) Now, the problem of placing the concept of topological group within the framework of the arithmetic of Boolean algebras with operators is that of expressing the continuity conditions. If $\sim$ designates the closure operator, then the continuity of $\smile$ is obviously given by $\tilde{x} \sim=\tilde{x} \smile$ for any $x$, and that of; is expressible as follows: for any $x, y, z$ if $(x ; y) \cdot z \neq 0$ then there are $u$ and $v$ such that $x \cdot \tilde{u}^{-} \neq 0, y \cdot \tilde{v}^{-} \neq 0$, and $\left(\tilde{u}^{-} ; \tilde{v}^{-}\right)+\tilde{z}^{-}=\tilde{z}^{-}$. Using this it follows $\tilde{x} ; \tilde{y}$ $\leqq(x ; y) \sim$ for any $x, y$, which easily gives that the closure of any subgroup is a subgroup as well as many other known properties. (Received July 19, 1954.)

\section{Alex Rosenberg and Daniel Zelinsky (p): Galois theory of con- tinuous transformation rings.}

A continuous transformation ring $L=L(M, N)$ is the ring of all continuous linear transformations on a pair of dual spaces $(M, N)$ over a division ring. A subring $A$ of $L$ is (strongly) Galois in case $A$ is the ring of invariants under a regular group $G$ of automorphisms of $L$ ("regular" defined as in Nakayama [Trans. Amer. Math. Soc. vol. 73 (1952) pp. 276-292]). Such an $A$ automatically satisfies (i) $A$ is a continuous transformation ring, hence has a socle, $S$; (ii) both $M$ and $N$ are completely reducible $A$-modules (i.e., $M S=M$ and $N S^{*}=N$ ) and the dimensions of $M$ and $N$ over $A$ are equal and finite; (iii) the centralizer of $A$ in $L$ is simple. Then the fundamental theorem of Galois theory holds for $L$ in the form: there is the usual 1-1 correspondence between the regular subgroups of $G$ and the subrings of $L$ that contain $A$ and satisfy (i), (ii), and (iii). Likewise, every isomorphism between two subrings of $L$ which is the identity on $A$ can be extended to an automorphism of $L$. These theorems are direct generalizations of the corresponding theorems of Nakayama [op. cit.] for the ring of all linear transformations on a finite-dimensional space. The proofs lean heavily on the methods and results of a paper by Rosenberg [Bull. Amer. Math. Soc. Abstract 60-4-471] and an unpublished book by N. Jacobson. (Received July 19, 1954.) 


\section{3t. Alex Rosenberg and Daniel Zelinsky: Extension of derivations in continuous transformation rings.}

We first give a simpler proof of the following theorem of Jacobson: Let $E$ be the ring of all endomorphisms of an abelian group $M$, and let $C$ be a primitive subring of $E$ with nonzero socle $S$ such that $M S=M$. Then any derivation of $C$ into $E$ can be extended to an inner derivation of $E$. Now let $L=L(M, N)$ be a continuous transformation ring, and let $C$ be a primitive subring of $L$ with nonzero socle $S$. Suppose further that $M S=M, N S^{*}=N$, and $S$ is contained in the socle of $L$. Then any derivation $\delta$ of $C$ into $L$ can be extended to an inner one of $L$ if either (i) $C$ is an algebra over the center $\Phi$ of $L$, the division ring of $C$ has finite dimension over $\Phi$, and $\delta$ is $\Phi$-linear; or (ii) there is a subring $A$ of $C$ annihilated by $\delta$, and whose centralizer in $E$ has a basis over the division ring of $L$ consisting of (not necessarily continuous) semilinear transformations. The latter is a generalization of a theorem of Nakayama [Duke Math. J. vol. 19 (1952) pp. 51-63]. (Received July 19, 1954.)

\section{4t. E. V. Schenkman: The splitting of certain solvable groups.}

Let $G$ be a finite group and let $H$ be the least member of the descending central series, that is, $G / H$ is nilpotent and $[G, H]=H$. Then if $H$ is Abelian: (1) there is a complement $X$ of $H$ in $G$, that is, $G=H X$, and $x \cap H=E$; (2) if $X$ and $Y$ are two complements of $H$ in $G$, then $X=h Y h^{-1}$ for some $h$ in $H$; (3) if $G$ has no center then $G$ and $A$ the group of automorphisms of $G$ are contained in the holomorph of $H$. (Received July 12,1954 .)

\section{Abe Sklar: A generalization of a summation formula of Ferrar.}

Ferrar [Compositio Math. vol. 4 (1937) pp. 394-405] has derived a class of summation formulae related to certain Dirichlet series, in particular the Riemann zetafunction. The author generalizes these and shows that the generalized formulae can be related to a wider class of Dirichlet series, including the Hecke-functions of signature $(\lambda, \kappa, \gamma)$. Some of the results of Apostol and Sklar [see Abstract 635] can be gotten as special cases of the generalized formulae. (Received July 19, 1954.)

\section{6t. Andrew Sobczyk: Canonical form for a real matrix.}

Any real nonsingular matrix $T$ may be reduced, by unimodular change of basis, to a diagonal block form $D_{1}, A_{1}, \cdots, D_{v}, A_{v}, H_{1}, B_{1}, \cdots, H_{w}, B_{w}$, where there is one pair of blocks $D_{h}, A_{h}$ for each real eigenvalue $t_{h}$, of multiplicity $p_{h}$, and one pair of blocks $H_{k}, B_{k}$ for each pair of conjugate complex eigenvalues $\left(x_{k} \pm i y_{k}\right)$, of multiplicity $q_{k}$. Let $r_{h}$ (at least one) be the number of independent eigenvectors which correspond to eigenvalue $t_{h}$; then block $D_{h}$ is of the form $t_{h} I$, where $I$ is the identity matrix of dimension $\left(r_{h}-1\right)$, and $A_{h}$ is a superdiagonal block of dimension $\left(p_{h}-r_{h}+1\right)$, having only one eigenvector $\tau_{h}$. Let $s_{k}$ be the number of pairs of conjugate eigenvectors $\xi_{1} \pm i \eta_{1}$, $\xi_{2} \pm i \eta_{2}, \cdots$, corresponding to eigenvalue $x_{k} \pm i y_{k}$, which are such that $\xi_{1}, \eta_{1}, \xi_{2}$, $\eta_{2}, \cdots$, are linearly independent in the real vector space $E_{n}$; then block $H_{k}$ has $\left(s_{k}-1\right)$ blocks of the form $\left(\begin{array}{c}x_{k} y_{k} \\ -y_{k} x_{k}\end{array}\right)$ on the diagonal, all other elements of $H_{k}$ being zeros; block $B_{k}$ has similar blocks on the diagonal, with all elements below the diagonal blocks being zeros. Any two nonconjugate eigenvectors of $B_{k}$ are complex linearly dependent. If $T$ is singular, of rank $m$, the canonical form is similar, with an additional diagonal block $Z$ which is superdiagonal with zeros on the diagonal, and of which the elements of the last $(n-m)$ rows are all zeros. (Received July 15,1954 .) 
667t. Irving Sussman: On rings in which $a^{n(a)}=a$. Preliminary report.

Rings characterized by the property that for each nonzero element not the identity, $a^{n(a)}=a, n(a)$ an integer exceeding 1 , and not necessarily fixed or bounded, form a subclass of the encompassing class of associate rings hitherto treated (Bull. Amer. Math. Soc. Abstracts 59-6-589 and 60-1-151). Rings belonging to this subclass are named simply periodic. Algebras of this type where $n(a)$ is bounded, or perhaps a fixed integer, have been treated under other names by several authors (see, for example, N. Jacobson, Algebraic algebras of bounded degree, Ann. of Math. vol. 46 (1945); also, where $n(a)$ is a fixed prime, see work by Foster, McCoy, Tarski, et al.). Every finite ring without proper nilpotents is simply periodic. Among other interesting properties developed for simply periodic rings: they necessarily have identities if there are non-zero-divisors, and each non-zero-divisor is a unit; the ring decomposes uniquely into local multiplicative groups, and is a field if and only if it contains no proper idempotent; it is necessarily finite if there are a finite number of idempotents; the ring is a certain composition of isomorphic Boolean rings; it is a subdirect sum of finite fields, etc. Other characterizations of these rings are given. It is shown by example that the theory is not pertinent to semigroups. (Received July 14, 1954.)

668t. Alfred Tarski: Some remarks and problems concerning isomorphism of algebras. Preliminary report.

The result (I) of the abstract of W. Hanf (Abstract 651) implies the following: (II) There are Boolean algebras $\mathfrak{A}$ and $\mathfrak{E}$ such that $\mathfrak{X} \times \mathfrak{A}$ and $\mathfrak{E} \times \mathfrak{E}$ are isomorphic while $\mathfrak{A}$ and $\mathfrak{E}$ are not. In fact, $\mathfrak{A}$ is the same as in (I), and we put $\mathfrak{E}=\mathfrak{A} \times \mathfrak{B}_{1}$, where $\mathfrak{B}_{1}$ is a two-element algebra. In topological translation (II) yields: (II') There is a compact 0-dimensional space $S$ with four subspaces $S_{1}-S_{4}$ such that (i) each of the subspaces $S_{1}-S_{4}$ is closed in $S$; (ii) $S$ is the union of $S_{1}$ and $S_{2}$ as well as of $S_{3}$ and $S_{4}$; (iii) $S_{1}, S_{2}$ are both disjoint and homeomorphic, and so are $S_{3}, S_{4}$; (iv) $S_{1}$ and $S_{3}$ are not homeomorphic. Using an argument of the author in his book Cardinal algebras, New York, 1949, p. $209 \mathrm{ff}$., the results (I) and (II) are carried over to commutative semigroups; in (I) one takes for $\mathfrak{B}_{1}$ and $\mathfrak{B}_{2}$ the additive semigroup of natural numbers. Open problems: (1) Are there denumerable Boolean algebras $\mathfrak{A}$ and $\mathfrak{E}$ satisfying the conditions of (II)? (2) Is it possible to extend (I) and (II) to groups and in particular to Abelian groups? (Received July 23, 1954.)

669. Leonard Tornheim: The inessential discriminant divisors of cubic and certain quartic fields.

The primes occurring in the inessential discriminant divisor of an algebraic number field of degree $n$ over the rational field are known to be less than $n$. When $n=3$, 2 occurs to at most the first power and a necessary and sufficient condition for this to happen expressed in terms of a defining equation is obtained by means of a minimal basis. For the quartic subfield of the field generated by a primitive $p$ th root of unity with $p$ a prime and $p \equiv 1(\bmod 4)$, Carlitz [Proc. Amer. Math. Soc. vol. $3(1952)$ pp. 688-692] determined when 2 and 3 appear. These also occur only to the first power. (Received July 19, 1954.)

\section{0t. A. D. Wallace: The topological invariance of ideals in mobs.}

Let $S$ be a compact connected mob (=Hausdorff semigroup) and let $G$ be an abelian group. Let the codimension of $S$ (relative to $G$ ) be at most $n$ and let $N$ be a 
closed set in $S$ with $N \subset_{t_{1}} N$ for some $t_{1} \in S$. Then (i) if $H^{n}(N, G) \neq 0$ the minimal ideal $K$ of $S$ is also a minimal right ideal; (ii) if $A$ is a floor for an element of $H^{n}(N, G)$, then $A$ is a left ideal contained in $K$. It follows that if also $N \subset N t_{2}$ for some $t_{2} \in S$, then $K$ is a floor for each element $H^{n}(N, G)$ and thus $K$ is a topological invariant of $S$. Moreover $K$ is a (topological) subgroup of $S$. If $N$ is a subgroup of $S$ with $H^{n}(N, G) \neq 0$, then $K=N$. (Received April 12, 1954.)

\section{1t. L. M. Weiner: The algebra of semi-magic squares.}

A square matrix $A=\left(a_{i j}\right)$ is called an $S$-matrix if $\sum_{i=1}^{n} a_{i j}=\sum_{i=1}^{n} a_{j i}=S(A)$ for each $j$. The set of all $S$-matrices of order $n$ forms a subalgebra of the total matric algebra of degree $n$. This algebra is the direct sum of the one-dimensional ideal $M$ consisting of all matrices $A$ for which $a_{i j}=a, a$ constant, and the $(n-1)^{2}$-dimensional ideal $N$ consisting of all matrices $A$ for which $S(A)=0$. A basis for the ideal $N$ is given by the matrices $A_{i j}(i, j=2,3, \cdots, n)$, where $A_{i j}$ is the matrix which has 1 in the first row and first column, -1 in the first row and $j$ th column, -1 in the $i$ th row and first column, and 1 in the $i$ th row and $j$ th column. (Received July 8,1954 .)

672. A. L. Whiteman: $A$ sum connected with the series for the partition function.

In the formula for the number $p(n)$ of unrestricted partitions of an integer $n$ there appears the sum (1) $A_{k}(n)=\sum \omega_{h, k} \exp (-2 \pi i h n / k)$, where $h$ ranges over those numbers which are less than $k$ and relatively prime to $k$. The numbers $\omega_{h, k}$ are certain $24 k$ th roots of unity which arise in the theory of modular functions. Some years ago Atle Selberg proved (but did not publish) the result that $A_{k}(n)$ may be expressed alternatively in the form (2) $A_{k}(n)=(k / 3)^{1 / 2} \sum(-1)^{l} \cos [(6 l+1) \pi / 6 k]$, where $l$ runs over integers in the range $0 \leqq l<2 k$ which satisfy the congruence $\left(3 l^{2}+l\right) / 2 \equiv-n$ $(\bmod k)$. In this paper Selberg's formula is proved by showing that $(1)$ is the finite Fourier series expansion of (2). Selberg's formula is also used to provide a new approach to the evaluation and factorization of the $A_{k}(n)$. (Received May 20,1954.)

673. L. R. Wilcox: An imbedding theorem for semi-modular lattices. Preliminary report.

Let $M$ be a complemented modular lattice of length $\geqq 3$ in which join and meet are $U, \cap$. Let $L \subset M$ satisfy (1) $0,1 \in L$; (2) $a, b \in L$ implies $a \bigcup b \in L$; (3) every $a, b \in L$ possess a g.1.b. $a b \in L$ with respect to elements of $L$; (4) if $a \in M, b \in L, a \leqq b$, then there exists $x \in L$ with $a \bigcup_{x=b, a} \cap x=0 . L$ is then called a regular subsystem of $M$. It is shown to be a left complemented lattice, i.e., in it modularity is a symmetric relation, and it possesses a strong complementation property (L. R. Wilcox, Bull. Amer. Math. Soc. vol. 48 (1942) pp. 453-457), though it need not be a sublattice of $M$ or modular. It is shown that under certain additional assumptions, primarily concerning complementation, an abstract left complemented lattice $L$ of length $\geqq 6$ may be imbedded into a complemented modular lattice $M$ so that $L$ is (isomorphically) a regular subsystem of $M$. The principal tools used in the imbedding are a general theory of independence and a theory of quasi-dual-ideals, i.e., subsets $S$ of $L$ satisfying (a) $x \in S, y \geqq x$ implies $y \in S$, and (b) if $z=x \bigcap y$ with $x, y \in S$ and $(x, y)$ modular, then $z \in S$. (Received July 9, 1954.)

\section{K. G. Wolfson: Annihilator rings.}

The structure of annihilator algebras (which generalize dual algebras) [Bonsall 
and Goldie, Proc. London Math. Soc. vol. 4 (1954) pp. 154-167] is generalized to arbitrary topological rings. Let $K$ be a topological ring in which the intersection of the closed regular maximal right ideals is zero, and which possesses the annihilator property $\left({ }^{*}\right)$ a left (right) ideal $J$ has zero right (left) annihilator if, and only if $\bar{J}=K$. Then $K$ is the closure of the direct sum of simple rings with the annihilator property. For each of the simple components $K_{\nu}$ there exists a pair of topological vector spaces $\left(A_{\nu}, B_{\nu}\right)$ over a topological division ring $D_{\nu}$, which are dual relative to a continuous "inner product" such that each space is the set of all continuous linear functionals on the other. Then $K_{\nu}$ is the closure of the ring of all finite-valued linear transformations on $A_{\nu}$ which have adjoints on $B_{\nu}$. In particular, a semi-simple discrete annihilator ring is a direct sum of simple rings with minimum condition, and is therefore a dual ring. The converse question of when dual spaces give rise to simple annihilator rings is examined. (Received July 19, 1954.)

\section{ANALYsis}

675. H. A. Antosiewicz: Stable systems of differential equations with integrable forcing term.

Vector equations $d x / d t=p(x, t)\left({ }^{*}\right)$, defined for all $x$ and $t \geqq 0$, are considered subject to perturbations (i) $q(x, t)$ where $\|q(x, t)\| \leqq\|x\| h(t)$ for $\|x\| \leqq \xi, t \geqq 0$, (ii) $q(x, t, z)$ where $\|q(x, t, z)\| \leqq \phi(z) h(t)$ for $\|x\| \leqq \xi, t \geqq 0$ with $\phi(z)$ continuous at $z=0$ and $\phi(0)=0$, $h(t)$ being a non-negative function with $\int_{0}^{\infty} h(t) d t=H<\infty$. Assuming that $\left({ }^{*}\right)$ possesses a Liapunov function $V(x, t)$, it is shown in case (i) that if $V(x, t)$ is a quadratic form in $x$ with uniformly bounded coefficients then every solution of the perturbed equation satisfies $\left\|x\left(t, x_{0}, t_{0}\right)\right\| \leqq M\left\|x_{0}\right\|$ for $t \geqq t_{0}+T$ : where $0<M<1, T\left(M, x_{0}\right)>0, x_{0}$ sufficiently small; and in case (ii) that, given any $\epsilon>0$, there exist constants $\xi(\epsilon, H)>0, \delta(\epsilon, H)>0$ such that every solution of the perturbed equation with $\|z\| \leqq \xi$ satisfies $\left\|x\left(t, x_{0}, t_{0}\right)\right\|$ $\leqq \epsilon$ for $t \geqq t_{0}$ whenever $\left\|x_{0}\right\| \leqq \delta$. (Received June 25,1954 .)

\section{Nachman Aronszajn: Coercive quadratic integro-differential} forms.

A finite number of partial differential operators $A_{k}$ of orders $\leqq m$ defined in a domain $G$ of the $n$-dimensional euclidean space, with continuous coefficients, are considered. Denote by $q_{k}(x ; \xi)$ the characteristic polynomial, homogeneous of order $m$, of $A_{k}\left(q_{k} \equiv 0\right.$ if $A_{k}$ is of order $\left.<m\right)$. For functions $u$ of class $C^{(m)}$ in $G$ and a domain $D$ with $\bar{D} \subset G$ put $\|u\|_{D}^{2}=\int_{D} \sum\left|A_{k} u\right|^{2} d x$. For $p=0,1, \cdots, m$ put $\|u\|_{p, D}^{2}=\sum \int_{D}\left|\partial^{\alpha} u\right|^{2} d x$, $\partial^{\alpha} u$ running through all partial derivatives of $u$ of order $\leqq p$. The quadratic form $\|u\|_{D}^{2}$ is called coercive in a domain $D^{\prime} \subset D$ if for some constant $c>0,\|u\|_{m, D^{\prime}}^{2} \leqq c\left(\|u\|_{D}^{2}\right.$ $+\|u\|_{m-1, D^{\prime}}^{2}$ ). In what follows, the boundary of $D^{\prime}$ is of class $C^{(m)}$. Theorem 1: If $\overline{D^{\prime}} \subset D,\|u\|_{D}^{2}$ is coercive in $D^{\prime}$ if and only if for each $x \in \overline{D^{\prime}}$ there is no common real null-vector $\xi \neq 0$ for all $q_{k}(x, \xi)$ (this is equivalent to ellipticity of $A=\sum A_{k}^{*} A_{k}$ in $\overline{D^{\prime}}$ ). Theorem 2: If $D^{\prime} \subset D$ and at every common boundary point $y$ of $D$ and $D^{\prime}$, the boundary of $D$ is locally of class $C^{(m)},\|u\|_{D}^{2}$ is coercive in $D^{\prime}$ if and only if the condition of Theorem 1 is satisfied and for every common boundary point $y$ there is no common complex nullvector $\xi \neq 0$ for all $q_{k}(y, \xi)$ with imaginary part orthogonal to the tangential hyperplane at $y$. These theorems allow one to develop a theory of differential problems for the operator $A=\sum A_{k}^{*} A_{k}$ with general boundary conditions in a similar way as Gårding's inequality does in the case of Dirichlet boundary conditions (see L. Gårding, C. R. Acad. Sci. Paris vol. 233 (1951) pp. 1554-1556; Math. Scand. vol. 1 
(1953) pp. 55-72; also F. Browder, Proc. Nat. Acad. Sci. U.S.A. vol. 38 (1952) pp. 230-235, 741-747). (Received July 19, 1954.)

\section{7t. R. G. Bartle: On compactness in functional analysis.}

S. Kakutani [J. Math. Soc. Japan vol. 1 (1951) pp. 228-231] has introduced the notion of the (strong) pairing of two abstract sets to obtain short, symmetric proofs of the Arzela-Ascoli compactness criterion in $C(X)$ and Schauder's well-known theorem on compact operators. The author introduces a corresponding notion of weak pairing to obtain corresponding weak compactness results. Among the applications are previously announced results of the author [Abstract 59-6-693] giving criteria for pointwise and weak compactness in $C(X)$, and extensions or simplifications of theorems due to Gantmacher, Gelfand, and Sirvint concerning [weakly] compact operators. This paper supersedes the one referred to above. (Received July 15, 1954.)

678. J. S. Bendat (p) and Seymour Sherman: Monotone and convex operator functions.

Charles Loewner [Über monotone Matrixfunctionen, Math. Zeit. vol. 38 (1934)] showed that analyticity plus the property of mapping the complex open upper halfplane into itself is characteristic for the class of monotone matrix functions of arbitrarily high order. This class coincides with the class of monotone operator functions, the operators being bounded self-adjoint operators in a Hilbert space. For convex operator functions, no characterization or relationship with monotone operator functions was known. The principal result in this paper establishes the proper correspondence between monotone and convex operator functions by showing that a convex operator function $f(x)$, i.e., for each scalar $\alpha, 0<\alpha<1, \alpha f\left(X_{1}\right)+(1-\alpha) f\left(X_{2}\right) \geqq f\left(\alpha X_{1}+(1-\alpha) X_{2}\right)$, is necessarily analytic, and if $f(x)=\sum_{1}^{\infty} a_{n} x^{n}$ in $|x|<R<\infty$, where $R$ is the radius of convergence, then $f(x)$ can be uniquely represented by a Stieltjes integral $f(x)=a_{1} x$ $+\int_{-1 / R}^{1 / R} x^{2}(1-t x)^{-1} d \psi(t)$, where $\psi(t)$ is a bounded nondecreasing function, continuous on the left, with $\psi(-1 / R)=0$. Further considerations then prove that $f(x)=\sum_{1}^{\infty} a_{n} x^{n}$ is a convex operator function in $|x|<R$ if and only if $f(x) / x$ is a monotone operator function in $|x|<R$. (Received June 15, 1954.)

679t. Stefan Bergman: On solutions of certain linear partial differential equations in three variables.

Using analytic functions which are orthonormal, when integrating over the distinguished boundary surface $D^{2}$ of a domain $M^{4}$, the author derives a formula expressing the values of an analytic function $f\left(z_{1}, z_{2}\right)$ of two complex variables in terms of its values on the distinguished boundary surface. Since the kernel $K$ of this system is finite, one obtains bounds for $|f|$ inside the domain $M^{4}$. The author introduces meromorphic functions which are orthogonal to $K$ in $M^{4}$. Using these functions he obtains new representations in $M^{4}$ for certain classes of meromorphic functions. Using integral operators which transform analytic functions of two complex variables into solutions $\chi(x, y, z)$ of $L(\chi) \equiv\left(\partial^{2} \chi / \partial x^{2}\right)+\left(\partial^{2} \chi / \partial y^{2}\right)+\left(\partial^{2} \chi / \partial z^{2}\right)+C\left(r^{2}\right) \chi=0$, where $C$ is an entire function of $r^{2}=x^{2}+y^{2}+z^{2}$ (see Trans. Amer. Math. Soc. vol. 68, $461 \mathrm{ff}$.), he obtains from the results mentioned above various theorems about the solutions $\chi$ of $L(\chi)=0$. (Received September 1, 1954.)

\section{Arthur Bernhart: Note on Fuchsian differential equations.}

For linear differential equations with analytic coefficients Fuchs obtains power 
series solutions, and shows convergence by comparison with a dominant equation. A modification by Günther simplifies the comparison solution at the expense of its radius of convergence. This paper combines the advantages of both existence proofs. (Received July 19, 1954.)

\section{W. W. Bledsoe (p) and A. P. Morse: Product measures.}

Suppose $\mu$ and $\nu$ are (outer) measures. Let $\theta \in$ Fubini $\mu \nu$ if and only if $\theta$ measures the product space in such a way that: $\iint f(x, y) \mu d x \nu d y=\int f(z) \theta d z=\iint f(x, y) \nu d y \mu d x$ whenever $f$ is $\theta$-summable; $A \times B$ is $\theta$-measurable and $\theta$-finite whenever $A$ is $\mu$-measurable and $\mu$-finite, $B$ is $\nu$-measurable and $\nu$-finite. Also let $\theta \in C$ lin $\Omega$ if and only if $\Omega$ is a topology and $\theta$ measures space $\Omega$ in such a way that: open sets (members of $\Re$ ) are $\theta$-measurable; open sets have a certain closed subset property; $\Omega$ is $\theta$-almost lindelof (no local compactness is assumed). Suppose $\mathfrak{M}$ and $\mathfrak{N}$ are topologies and $\mathfrak{I}$ is their topological product. A new (topology free) product measure $\phi$ is defined which allows more freedom of action than the classical product measure and which belongs to Fubini $\mu \nu$. For $\mu \in$ Clin $\mathfrak{M}, \nu \in C$ lin $\mathfrak{N}$, it is proved that $\phi \in C$ lin $\mathfrak{T}$, from which it follows (since $\phi \in$ Fubini $\mu \nu$ ) that $\iint f(x, y) \mu d x \nu d y=\int f(z) \phi d z=\iint f(x, y) \nu d y \mu d x$ whenever $f$ is a $\mathfrak{I}$-Borelian function which is dominated by a $\phi$-summable function. Other product measures are defined which preserve more restrictive hypotheses on $\mu$ and $\nu$. Preliminary to the principal results are discussions of integrable functions and closed subset properties of topological measures. (Received June 2,1954.)

\section{2t. H. D. Block: Perturbations of nonlinear eigenvalue problems.}

In a paper to appear shortly, P. C. Rosenbloom has considered the eigenvalue problem for a perturbed operator [cf. Bull. Amer. Math. Soc. Abstract 58-4-440]; i.e. let $T_{0}$ be a linear operator on a Banach space with $T_{0} x_{0}=\lambda_{0} x_{0}$. If $U$ is a "small" operator what can be said about the solutions, $(x, \lambda)$, of the equation $\left(T_{0}+U-\lambda I\right) x$ $=0$ ? We use the methods introduced by Rosenbloom to deal with this problem when $T_{0}$ is not linear, and $U=U(\lambda)$ is not linear. It is proved that, under suitable conditions, the solution $(x, \lambda)$ is unique and lies close to $\left(x_{0}, \lambda_{0}\right)$. An iterative method for obtaining the solution is given as well as numerical estimates of the rate of convergence and the errors of terminating the iteration after a finite number of steps. (Received July 19, 1954.)

\section{3t. R. P. Boas, Jr.: Moments of analytic functions.}

The author proves the following generalization of results of San Juan [C. R. Acad. Sci. Paris vol. 236 (1953) pp. 1941-1943] and Sunyer Balaguer [Revista Mat. Hisp.Amer. (4) vol. 13 (1953) pp. 241-246]. If $f(z)$ is analytic and of exponential type for $x \geqq 0$ and is bounded on the imaginary axis, and if $\int_{0}^{\infty}\left|f\left(r e^{i \theta}\right)\right| r^{n} d r<n^{n} e^{-n \phi(n)}, \phi(n) \rightarrow \infty$, for some $\theta(-\pi / 2<\theta<\pi / 2)$ and for an infinity of $n$, then $f(z) \equiv 0$. The theorem is deduced from a general result of Ahlfors and Heins [Ann. of Math. (2) vol. 50 (1949) pp. 341-346]. (Received June 1, 1954.)

684. H. D. Brunk, G. M. Ewing (p), and W. T. Reid: The minimum of a certain definite integral suggested by the maximum likelihood estimate of a distribution function.

For $a \geqq 0, b \geqq 0,0 \leqq u \leqq 1$, define $L(u ; a, b)=-a \ln u-b \ln (1-u)$ if $0<u<1$. Let $g(x)$ be bounded nondecreasing and not constant on $[0, X]$, let $\mu$ be the LebesgueStieltjes measure generated by $g$, and let $\alpha(x)$ be $\mu$-measurable on $[0, X]$. After suit- 
ably extending $L(u ; a, b)$, the function $F(u ; x)=L[u ; \alpha(x), 1-\alpha(x)]$ is defined finite or $\infty$ for $0 \leqq u \leqq 1,0 \leqq x \leqq X$. Let $\mathfrak{M}$ be the class of nonincreasing functions on $[0, X]$ bounded by 0 and 1 and consider $J[p] \equiv \int_{[0, X]} F[p(x) ; x] d \mu$. The principal results of this paper are (i) that there exists $P$ minimizing $J$ in $\mathfrak{M}, P$ being unique except for sets of $\mu$-measure zero, (ii) that if $P$ minimizes $J$ in $\mathfrak{M}$ then, almost everywhere $(\mu), P$ is equal to inf $0 \leqq u \leqq x ~ \sup _{x \leqq 0 \leqq x} M(I)$, and to $\sup _{x \leqq v \leqq X} \inf _{0 \leqq u \leqq x} M(I)$, where $x \in I$, an interval with end points $u, v$, and $M(I)$ is the integral mean of $\alpha(x)$ over $I$ with respect to $\mu$. (Received July 19, 1954.)

\section{5t. Paul Civin: Abstract Riemann sums.}

A theorem of Jessen [Ann. of Math. vol. 35 (1934) pp. 248-251] asserts for $f(x)$ Lebesgue integrable on $[0,1]$ and of period one the almost everywhere existence of $\lim _{n \rightarrow \infty} 2^{-n} \sum_{k=0}^{2^{n-1}} f\left(x+k 2^{-n}\right)=\int_{0}^{1} f(t) d t$. The theorem is shown to be a special case of a theorem analogous to the Birkhoff ergodic theorem asserting the almost everywhere limits of sums $2^{-n} \sum_{k=0}^{2^{n}-1} f\left(T^{k / 2^{n}} x\right)$ on a $\sigma$-finite measure space. Analogues are also obtained for the ergodic theorems of Hopf and Hurewicz [Ann. of Math. vol. 45 (1944) pp. 192-196]. (Received May 28, 1954.)

\section{6t. Paul Civin: Some ergodic theorems involving two operators.}

A version of the Hurewicz ergodic theorem [Ann. of Math. vol. 45 (1944) pp. 192$206]$ is obtained for two operators $T$ and $U$ on a $\sigma$-finite measure space. Each operator is assumed to be a 1-1 transformation of the space onto itself and to satisfy the Birkhoff ergodic theorem. As a corollary the Hopf ergodic theorem is carried over to the form that $\lim _{n \rightarrow \infty} \sum_{k=0}^{n} f\left(T_{x}^{k}\right) / \sum_{k=0}^{n} g\left(U_{x}^{k}\right)$ exists almost everywhere when $T$ and $U$ are measure preserving, $f(x)$ integrable and $g(x)>0$. An extension is thus made of a result of Harris and Robbins (Proc. Nat. Acad. Sci. U.S.A. vol. 39 (1953) pp. 860-864) concerning Markov chains admitting an infinite invariant measure. (Received May 28,1954 .)

687. K. L. Cooke: Forced periodic solutions of a stable nonlinear differential-difference equation.

In this paper it is proved that the differential-difference equation $u^{\prime}(t+1)=a u(t)$ $+b u(t+1)+f(u(t), u(t+1), \omega t)+k g(\omega t)$ has a continuous, asymptotically stable, periodic solution, of period $1 / \omega$, provided $|k| /(1+\omega)$ is sufficiently small, and provided the following conditions are satisfied: (1) all the roots of $z e^{z}-b e^{z}-a=0$ have negative real parts; (2) $a, b, k$, and $\omega$ are real numbers, $\omega>0$, and $t$ is a real variable; (3) $g(t)$ is real and continuous for all $t$, has period one, and the average of $g(t)$ over a period is zero; (4) $f(x, y, t)$ is real and continuous for all $(x, y, t)$, has period one in $t, f(0,0, t)$ $=0$, and for any $\epsilon>0,|f(x, y, t)-f(\xi, \eta, t)| \leqq \epsilon(|\xi-x|+|\eta-y|)$ for small enough $|\xi-x|$ and $|\eta-y|$, uniformly in $t$. The periodic solution is constructed by the method of successive approximations. The method may be extended so as to be applicable to systems of differential-difference equations. (Received June 14, 1954.)

688. Philip Davis and H. O. Pollak (p): Complex biorthogonality for certain sets of polynomials.

Let $\left\{L_{n}\right\}$ be a set of linear functionals complete in the set of analytic functions square-integrable over a complex domain $B$. A basic theorem of Walsh and Davis (In terpolation and orthonormal systems, Journal d'Analyse Mathématique vol. 2 (1952) pp 1-28) states that there exist linear combinations $L_{n}{ }^{*}$ of $L_{n}$, and functions $\phi_{n}{ }^{*}$, such that 
$L_{n}{ }^{*}\left(\phi_{m}{ }^{*}\right)=\delta_{m n}$ and $\iint_{B} \phi_{n}{ }^{*} \phi_{m}^{*} d A=\delta_{m n}$. The present paper extends this theorem and applies it to the development of a unifying theory for different sets of polynomials and rational functions. As examples, an $L^{2}$-theory is derived from this unified point of view for Tschebysheff polynomials of the second kind, Takenaka-Walsh interpolation rationals for the unit circle, and Faber-type polynomials for an arbitrary domain $B$ with analytic boundary. (Received July 19, 1954.)

689t. M. M. Day: On smoothing normed spaces. Preliminary report.

A result of Klee (Trans. Amer. Math. Soc. vol. 74 (1953) pp. 10-43, A1.7) which applies only to reflexive spaces is improved as follows: Let $B^{\prime}$ be a smooth reflexive space and let $T$ be a linear operator carrying $B^{\prime}$ into a dense subset of a normed space $B$; then $B$ is isomorphic to a smooth space $B_{1}$ and $B_{1}{ }^{*}$ is strictly convex. This lemma proves smoothability of $c_{0}(I)$, the space of functions vanishing at infinity on an arbitrary index set $I$, when $B^{\prime}$ is taken to be $l_{2}(I)$, and of $L_{1}(\quad)$, the space of summable functions on a space of finite measure, when $B^{\prime}$ is taken to be $L_{2}(\quad$ ). (Received July 19, 1954.)

690. W. F. Donoghue: A theorem concerning bounded linear operators on reflexive Banach spaces.

If $T$ is a bounded linear transformation of norm $\leqq 1$ on a reflexive infinite-dimensional Banach space, there exists no element $X$ in the space such that the closed circled convex hull of the sequence $\left\{T^{n} X\right\}, n \geqq 0$, contains an interior point. That the hypothesis of reflexivity is needed is shown by an example involving the space $l^{\prime}$. (Received September 2, 1954.)

\section{1t. R. J. Duffin: Some properties of discrete analytic functions.}

Complex-valued functions are considered which are defined at the Gaussian integer points of the complex plane. The Gaussian integer points form a lattice which breaks up the plane into squares. Jacqueline Ferrand defined a function to be "analytic" at one of these squares if the difference quotient across one diagonal is equal to the difference quotient across the other diagonal. She found several interesting analogies between such discrete analytic functions and ordinary analytic functions. In this paper analogues of the Cauchy integral formula are developed. The line integral is, of course, replaced by a suitable summation over the lattice points. The explicit evaluation of the Cauchy kernel is found. Asymptotic development of this kernel is furnished by methods previously developed by the writer and D. H. Shaffer. Functions are considered which are bounded and analytic in the upper half-plane; the real and imaginary parts on the real axis are related by an analogue of the Hilbert transform. Expansions in terms of polynomials and other type functions are considered. (Received July 20,1954 .)

692. Paul Erdös, Fritz Herzog, and George Piranian (p): On polynomials whose zeros lie on the unit circle.

Let (1) $P(z)=\prod_{i=1}^{n}\left(z-z_{j}\right)$, where $\left|z_{j}\right|=1$ for $j=1,2, \cdots, n$. It is easily seen that there exists a continuous curve $\Gamma$, starting at the origin and ending at a point of the unit circle, such that $|P(z)|<1$ at all points of $\Gamma$ except the origin; an analogous statement holds for the inequality $|P(z)|>1$. However, C. Loewner found a complicated example (unpublished) of a polynomial (1) for which $|P(z)|>1$ on at least one point of every radius of the unit disc. In the present paper is exhibited a simple 
example of a polynomial (1) with the property that on each radius of the unit disc there exist two points at which $|P(z)|>1$ and $|P(z)|<1$, respectively. It is also shown that, for each polynomial (1) of degree not greater than 4 , there exists a radius of the unit disc on which the inequality $|P(z)|<1-|z|^{n}$ holds everywhere. (Received July 19, 1954.)

693. Paul Erdös, Fritz Herzog (p) and George Piranian: Sets of divergence of Taylor series and of trigonometric series.

In two previous papers (Duke Math. J. vol. 16 (1949) pp. 529-534 and vol. 20 (1953) pp. 41-54) Herzog and Piranian have shown that (a) if a set on the unit circle $C$ is of type $G_{\delta}$, it is the set of divergence of a Taylor series and (b) certain sets on $C$, not of type $G_{\delta}$, are sets of divergence of Taylor series whose partial sums are uniformly bounded on $C$. In the present paper, the authors use certain polynomials, introduced by Fejér, to obtain the following extensions of these results: (i) If a set on $C$ is of type $F_{\sigma}$ and of logarithmic measure zero, it is the set of divergence of a Taylor series whose partial sums are uniformly bounded on $C$ and which represents a function continuous on $|z| \leqq 1$; (ii) If a set on $C$ is the union of a set $E_{1}$ of type $G_{\delta}$ and a set $E_{2}$ of logarithmic measure zero and of type $F_{\sigma}$, it is the set of divergence of a Taylor series. Analogues to (i) and (ii) are proved for Fourier series and trigonometric series, respectively. The analogue to (i) is a generalization of a result due to Tandori (Publ. Math. Debrecen vol. 2 (1952) pp. 191-193). (Received May 28, 1954.)

\section{4t. Evelyn Frank: On the calculation of the roots of equations.}

The Newton method for the calculation of the solution of equations with $n$ unknowns is here extended to include not only the linear terms of the Taylor series expansions but also terms of higher degree. Remarkably simple formulas are obtained for equations in two variables. These are exceptionally convenient for use in the computation of the complex roots of an equation $F(z)=0$. In this connection, a novel variation of Horner's method is presented for the computation of partial derivatives of rational integral functions. The convergence of the method to the value of the solution is considered, and an estimate of the error involved in the use of only a certain number of terms is indicated. A new method is also presented for the computation of bounds for the roots. (Received February 16, 1954.)

695. W. B. Fulks: $A$ note on the steady state solutions of the heat equation.

Let $R$ be a bounded region whose boundary $C$ is a simple closed curve with continuous curvature. Then the unique bounded solution for continuous $\phi(x), \psi(x, t)$ of the boundary value problem $\Delta u(x, t)-\partial u / \partial t=0, x \in R, t>0 ; u(x, 0)=\phi(x), x \in R$; $u(x, t)=\psi(x, t), x \in C, t>0$ is given by $u(x, t)=\int_{R} G_{R}(x, y, t) \phi(y) d A_{y}+\int_{0}^{t} \int_{C} H_{R}(x, y, t-s)$ $\psi(y, s) d C_{y} d s$ where $G_{R}$ and $H_{R}$ are the Green's functions for the region $R$. The author uses this formula to show that if $\phi(x)=0, \psi(x, t)=\psi(x) \geqq 0$ is independent of time, then the solution $u(x, t)$ is a monotone increasing function of $t$ which converges uniformly to the solution of the boundary value problem $\Delta u(x)=0, x \in R ; u(x)=\psi(x)$, $x \in C$, as $t \rightarrow \infty$. (Received July 19, 1954.)

696. Michael Golomb: On the mean-square approximation of functions of several variables by products of functions of fewer variables.

The existence of functions $u(x), v(y), w(z) \in L_{2}$ which minimize the functional 
$\iiint[f(x, y, z)-u(x) v(y) w(z)]^{2} d x d y d z$ for given $f(x, y, z) \in L_{2}$ is proved and it is shown that $u, v, w$ are the characteristic solutions of a certain triple of nonlinear integral equations belonging to its largest characteristic value. Furthermore, the function $U(x, y)=u(x) v(y)$ is the characteristic solution of a single nonlinear integral equation belonging to its largest characteristic value. Conditions are derived under which the simpler problem of minimizing $\iiint[f(x, y, z)-U(x, y) W(z)]^{2} d x d y d z$ has the solution $U(x, y)=u(x) v(y), W(z)=w(z)$ of the above problem. Similar results are obtained for the functional $\iiint\left[f(x, y, z)-\sum_{k=1}^{n} u_{k}(x) v_{k}(y) w_{k}(z)\right]^{2} d x d y d z$ and for the corresponding variational problems in more than three independent variables. (Received July 19, 1954.)

697t. E. C. Gras: Generalized axially symmetric potential theory and the Weber-Schafheitlin discontinuous integrals.

Let $I=y^{r+\mu} b^{r+\nu} \int_{0}^{\infty} e^{-x t} J_{r+\mu}(y t) J_{r+\nu}(b t) t^{-(\mu+\nu+1)} d t$, where $r>0, \mu+\nu+1>-1$. Lim $I$ as $x \rightarrow 0^{+}$and $x^{-1}(y-b) \rightarrow \cot \beta$ is found explicitly by using the representation of a Bessel function by Sonine's integral (Watson, Theory of Bessel functions, p. 373), and by using the formula for the stream function of a "ring of sources" (A. Weinstein, Trans. Amer. Math. Soc. vol. 63 (1948) pp. 342-354). These limits for real values of $\mu$ and $\nu$ are extensions of previous results which were valid only for integers (E. C. Gras, On a generalization of the integrals of Weber and Schafheitlin, Bull. Amer. Math. Soc. Abstract 60-4-411). (Received May 20, 1954.)

\section{W. T. Guy, Jr.: A generalized Laplace transform.}

A "generalized Laplace" transform is defined using one of Meyer's $G$-functions as a kernel. The inversion formula is developed and a few examples are given. Varma's transform is obtained as a special case. (Received July 20,1954.)

699. Guy Johnson, Jr.: Collective singularities of families of analytic functions.

Let $F$ denote a family of functions $f(z)=\sum_{n=0}^{\infty} a_{n}^{f}\left(z-z_{0}\right)^{n}$. If there is a neighborhood of $z_{0}$ in which each function is holomorphic and $F$ is normal, then $z_{0}$ is called a regular point. Otherwise it is called a singular point of $F$. In particular if there is a neighborhood in which each function is holomorphic but none in which $F$ is normal, then $z_{0}$ is called a collective singularity. $R$ is called the radius of regularity of $F$ at $z_{0}$ if it is the largest number such that each function is holomorphic and $F$ is normal in $\left|z-z_{0}\right|<R$. In the case where $F$ is a bounded family, Mandelbrojt stated the formula $\lim \sup _{n \rightarrow \infty}$ l.u.b.f $\left|a_{n}^{f}\right|^{1 / n}=1 / R$. If $F$ is locally bounded in $\left|z-z_{0}\right|<R$ and for each function there is a sequence of integers $\left\{\lambda_{p}^{f}\right\}$ such that $n \neq \lambda_{p}^{f}, p=1,2, \cdots$, implies $a_{n}^{f}=0$ and $\lambda_{p+1}^{f} / \lambda_{p}^{f} \geqq \lambda>1$ where $\lambda$ is fixed for $F$, then each point on $\left|z-z_{0}\right|$ $=R$ is a singular point. Let $G$ denote a family of functions $g(w)$ which has radius of regularity $R=\infty$ and is bounded in any finite domain. Let $M_{g}(r)=\max |w|-r|g(w)|$, $M(r)=$ l.u.b.g $M_{g}(r)$ and $\lim \sup _{r \rightarrow \infty}(\log M(r)) / r=A$. If $A<\pi / e$, then the family $F$ of functions $f(z)=\sum_{n=0}^{\infty} g(n) z^{n}$ is normal in the complement of $\{z|| \log z \mid \leqq A e$, $\left.|z| \geqq e^{-a}\right\}$. (Received July 19, 1954.)

700t. Guy Johnson, Jr.: Regions of flatness for analytic functions and their derivatives.

The following definition and properties of regions of flatness extends those given by Mandelbrojt and Ulrich (Duke Math. J. vol. 18, pp. 549-556) from the special 
case of circles to simply-connected domains. Let $D_{\alpha}$ denote a simply-connected domain in the finite $z$-plane which contains $z=\alpha$. Let $\omega_{\alpha}(w)$ map the circle $|w|<1$ conformally on $D_{\alpha}$ such that $\omega_{\alpha}(0)=\alpha$ and $\omega^{\prime}(0)>0$. Then $\omega_{\alpha}(w)$ maps $|w|<\eta<1$ on a domain $D_{\alpha, v}$. Let $\mathcal{S}$ be an infinite set of domains $D_{\alpha}$ contained in a domain in which $f(z)$ is holomorphic and suppose $f(z) \neq 0$ in $D_{\alpha, \eta}$ except for at most a finite number of domains depending on $\eta$. If the family of functions $F_{\alpha}(w)=f\left[\omega_{\alpha}(w)\right]$ is normal in $|w|<1$, then the domains $D_{\alpha}$ will be called regions of flatness for $f(z)$. Suppose $f^{\prime}(z)$ $\neq 0$ in all but a finite number of domains $D_{\alpha, \eta}$. If there exist three positive constants $A, B$ and $C$ such that (1) $\omega_{\alpha}^{\prime}(0)\left|\left[\log _{2} f(\alpha)\right]^{\prime}\right|>A$ and (2) $\log |f(\alpha)|>\max \left\{B \omega_{\alpha}^{\prime}(0), C\right\}$ for all but a finite set of numbers $\alpha$, then the domains $D_{\alpha}$ are regions of flatness for $f^{\prime}(z)$. If $\mathcal{S}$ is denumerable, $f(z)$ tends to infinity in the domains $D_{\alpha, \eta}$ and (2) is replaced by $\log |f(\alpha)|>\max \left\{B \omega_{\alpha}^{\prime}(0), C / \omega_{\alpha}^{\prime}(0)\right\}$ then the domains $D_{\alpha}$ are regions of flatness for $f^{(k)}(z), k=1,2, \cdots$. (Received July 19, 1954.)

\section{V. L. Klee: Boundedness and continuity of linear functionals.}

This paper (which will appear in the Duke Math. J.) establishes connections between continuity of linear functionals and their boundedness on certain sets. (A) If $E$ is a metric linear space and $C$ is a convex subset of $E$, the following statements are equivalent: (i) There is a closed linear subspace $L$ of finite deficiency in $E$ such that $C \subset L$ and $C-C$ has nonempty interior relative to $L$. (ii) Every additive functional on $E$ which is bounded on $C$ is continuous on $E$. (B) If, in addition, either $E$ is a Banach space and $C$ is closed or $B$ is complete and $C$ is a closed cone, then (i) and (ii) are equivalent to (iii): Every additive functional on $E$ which is unilaterally bounded on $C$ is continuous on $E$. The second part of (B) includes a rather general result on continuity of non-negative additive functionals, from which it can be deduced that for $0<r<1$ the space $L^{r}$ admits no nontrivial non-negative additive functional, and that the algebra $L^{\omega}$ admits no nontrivial multiplicative additive functional. (Received July 6,1954 .)

\section{2t. D. C. Kleinecke: A generalization of complete continuity.}

It remains an unsolved problem whether the completely continuous operators on a general Banach space can be approximated in norm by operators with finite-dimensional ranges. In a sense the approximable operators are a lower "bound." An upper "bound" is found in the set of hyperfinite operators, that is the set of operators which map into the radical of the quotient algebra of the algebra of all operators by the ideal of approximable operators. This set can be characterized as the largest ideal of operators which have spectra of the same type as the completely continuous operators. The weakly completely continuous operators (Dunford and Pettis, Trans. Amer. Math. Soc. vol. 54 (1943) pp. 185-219) show the extension to hyperfinite operators is not trivial. It is shown that hyperfinite operators possess most of the interesting properties of the completely continuous operators. (Received May 14, 1954.)

\section{3t. D. C. Kleinecke: The essential and companion spectra.}

The essential spectrum $\sigma_{\infty} T$ of an operator $T$ is now defined as the spectrum of $T^{\prime}$, the image of $T$ in the quotient algebra by the approximable (or completely continuous) operators. (Compare Schwartz, Bull. Amer. Math. Soc. Abstract 60-2-270.) The companion spectra $\sigma_{\nu} T$ are the sets of $\lambda$ for which $\alpha(\lambda I-T)=\nu(\nu= \pm 1, \pm 2, \cdots)$ where $\alpha$ is the index (Yood, Duke Math. J. vol. 18 (1951) pp. 599-612). The zero spectrum $\sigma_{0} T$ is made up of two parts: $\sigma_{x} T$, spectral points $\lambda$ for which $\alpha(\mu I-T)=0$ 
and $\mu \in \sigma T$ for all $\mu$ in some neighborhood of $\lambda ; \sigma_{m} T$, isolated eigenvalues of finite multiplicity. $\Phi T$ is the union of $\sigma_{m} T$ and the resolvant set $\rho T$. It is shown that $\sigma_{\nu} T(\nu= \pm 1, \pm 2, \cdots), \sigma_{x} T, \Phi T$ are disjoint open sets whose boundaries lie in closed set $\sigma_{\infty} T$. If $P$ is hyperfinite (which includes completely continuous) then the essential and companion spectra are preserved, $\sigma_{\nu} T=\sigma_{\nu}(T+P)(\nu= \pm 1, \pm 2, \cdots, \infty)$; a component of $\Phi T$ may go into a component of $\sigma_{x} T$ and conversely. In particular, if $\sigma_{\infty} T$ does not divide the plane, $\Phi T=\Phi(T+P)$. (Received May 14, 1954.)

\section{4t. D. C. Kleinecke: The spectrum as a function.}

The correspondence $T \rightarrow \Phi T$ of elements of a Banach algebra to their spectra may be considered as a function from the algebra to the bounded closed subsets of the complex plane taken with the Hausdorff metric. Newburgh (Duke Math. J. vol. 18 (1951) p. 165-176) investigated the question of continuity for this function. He left open the question when the algebra has the uniform topology. The following example shows $\sigma$ is sometimes discontinuous in this case: Consider Hilbert space with base $e_{n}(n=0, \pm 1, \pm 2, \cdots)$; let $A e_{n}=-e_{n-1}$ and $P=\left(\cdot, e_{1}\right) e_{0}$. Then $A+\delta P \rightarrow A+P$ as $\delta \rightarrow 1$, but $\sigma(A+\delta P)=\{z|z|=1\}$ if $\delta \neq 1$, while $\sigma(A+P)=\{z|z| \leqq 1\}$. It is shown that a similar disappearance of a component of the resolvant set occurs in connection with every discontinuity of $\sigma$. (Received May 14, 1954.)

\section{5t. Jacob Korevaar: Analytic functions bounded in an angle and nice on a ray.}

The author assumes that $h \in C_{\rho}(\beta)$ (where $\rho$ is real, $\beta>0$ ) if (i) $h(z) \in B(\beta)$, that is, $h(z)=h\left(r e^{i \theta}\right)$ is analytic in $|\theta|<\beta$ while for every $\beta^{\prime}<\beta$ there exist numbers $A>0, a$ and $b$ such that $\left|h\left(r e^{i \theta}\right)\right|<A r^{-a}(1+r)^{b}$ in $|\theta| \leqq \beta^{\prime}$; (ii) $h(x) \in D_{\rho}$, that is, there exist numbers $B, C$ such that $\left|h^{(k)}(x)\right| \leqq B C^{k} k^{\rho k}(0<x<1, k=0,1, \cdots)$. Various inequalities are derived, showing among other things that if $h \in C_{\rho}(\beta)$ then $h\left(x e^{i \theta}\right) \in D_{\rho}$ for every $\theta,|\theta|<\beta$. The following characterization theorem is proved. $h \in C_{\rho}(\beta)$ if and only if there exists a function $H$ with the following properties. (i) $H(w)$ is analytic in some half-plane $u=\operatorname{Re} w<\gamma_{0}$; (ii) for every $\beta^{\prime}<\beta\left(\beta^{\prime}>0\right)$ there are $b_{1}, b_{2}$ and $\gamma<\gamma_{0}$ such that $\log |H(u+i v)| \leqq b_{1}+b_{2}|u|+\rho|u| \log |u|+\left(\pi / 2-\beta^{\prime}\right)|v|(u \leqq \gamma$, $-\infty<v<\infty)$; (iii) for every such $\gamma$ which is not an integer $\leqq 0$ one has $h(z)=P(z)$ $+(2 \pi i)^{-1} \int H(w) \Gamma(w) z^{-w} d w$, where the integral is taken along the line $u=\gamma$, and where $P$ is a polynomial of degree $<-\gamma(P=0$ if $\gamma>0)$. One consequence is that for $\beta>(1+\rho) \pi / 2$ (and $\beta>0$ ) there are no functions in $C_{\rho}(\beta)$ other than polynomials. Applications are made to the case where $h(z)$ is of the form $\sum a_{n} \exp \left(-n^{\lambda} z\right)(\lambda>0)$. Then (formally) $H(w)=\sum a_{n} n^{-\lambda w}$. (Received July 16, 1954.)

706t. Jacob Korevaar: Numerical Tauberian theorems for power series and Dirichlet series.

Throughout this paper the $a_{n}$ satisfy the Tauberian condition $a_{n} \geqq-n^{-1} \phi(n)$ $(n=1,2, \cdots)$, where $\phi(n)=A>0$ or $\phi(n)=A n^{\alpha}$ (or $\left.\phi(n)=n^{\alpha} L(n)\right)$. The above characterization of $C_{\rho}(\pi / 2)$ is used to show that the following two assertions are equivalent $(\lambda, \rho>0)$. (a) The series (1) $\sum a_{n} \exp \left(-n^{\lambda} x\right)$ converges when $x>0$ and its sum $f_{\lambda}(x)$ is in $D_{\rho}$. (b) The series $\sum a_{n} \exp \left(-n^{\mu} x\right)$ with $\mu=\lambda / \rho$ converges when $x>0$ and its $\operatorname{sum} f_{\mu}(x)$ is in $D_{1}$. For such functions $f_{\mu}(x)$ analytic at $x=0$, or rather, for corresponding integrals, Tauberian theorems may be proved by the methods of previous papers [Nederl. Akad. Wetensch. Proc. Ser. A vol. 57 (1954) pp. 36-45 and 46-56]. Various results for series (1) and series $\sum a_{n} n^{-w}$ follow. Example: let the series (1) 
converge when $x>0$ and let $f_{\lambda}(x) \in D_{\rho}(\rho>0)$. Then one has the estimate (2) $\left|s_{n}-s\right|$ $\leqq B n^{-\lambda / \rho} \phi(n)(n=1,2, \cdots)$, where $s=\lim f_{\lambda}(x)$ as $x \downarrow 0, s_{n}=\sum_{1}^{n} a_{k}$. (2) is best possible when $\lambda \leqq \rho$. When $\lambda>\rho$ one has the estimate $\left|s_{n}-s\right| \leqq C \exp \left(-\epsilon n^{\lambda / \rho}\right)$ $(n=1,2, \cdots ; \epsilon>0)$, a result related to gap series results. Several recent Tauberian theorems of the author can be brought within the present framework. Illustration: if the series (1) converges when $x>0$ and if $\left|f_{\lambda}(x)-s\right| \leqq E \exp \left\{-F x^{-1 /(\rho-1)}\right\}(0<x<\infty$, $F>0, \rho>1$ ), then $f_{\lambda}(x) \in D_{\rho}$. (Received July 16,1954 .)

\section{7t. Jacob Korevaar: Numerical Tauberian theorems for Lambert series and the Riemann hypothesis.}

Assuming the truth of the R.H. that $\zeta(s)$ has no zeros for $\sigma=\operatorname{Re} s>1 / 2$ one has the following Tauberian theorem. Let the Lambert series $\sum a_{n} n x /\left(e^{n x}-1\right)$ be convergent for $x>0$, let its $\operatorname{sum} f(x)$ be in $C_{\rho}(\pi / 2)$, and for every $\delta>0$ let $\left|a_{n}\right| \leqq A(\delta) n^{\delta-1}$ $(n=1,2, \cdots)$. Denote $\sum_{1}^{n} a_{k}$ by $s_{n}, \lim f(x)(x \downarrow 0)$ by $s$. Then for every $\epsilon>0$ one has an estimate $\left(^{*}\right)\left|s_{n}-s\right| \leqq B(\epsilon) n^{-c+\epsilon}(n=1,2, \cdots)$ where $c=1 / 2$ in the case $\rho<1$, $c=1 /(\rho+2)$ in the case $\rho \geqq 1$. The estimate $\left(^{*}\right)$ with $c=1 / 2$ holds whenever $\sum_{d / n} d a_{d}$ $=O\left(n^{-1 / 2+\eta}\right)$ for every $\eta>0$, and it might well hold in the case $\rho=1$. The above Tauberian theorem conversely implies the truth of the R.H. This follows from the example $a_{n}=\mu(n) / n: f(x)=x e^{-x} \in C_{0}(\pi / 2)$. In this case $\left(^{*}\right)$ gives $\left|\sum_{1}^{n} \mu(k) / k\right|$ $\leqq B(\epsilon) n^{-1 / 2+\epsilon}$, which implies that the Dirichlet series for $1 / \zeta(s)$ converges when $\sigma>1 / 2$. (Received July $16,1954$. )

\section{G. L. Krabbe: On the logarithm of uniformly bounded operators.} Preliminary report.

Let $V$ be a uniformly bounded operator in a reflexive Banach space, i.e. $\sup _{n}\left\|V^{n}\right\|$ $<\infty$. In this paper a study is made of the operator solutions of $\exp (i X)=V$. Theorem I: If neither +1 nor -1 is an eigenvalue of $V$, then there is at most one bounded solution $A$ with spectrum on the closed interval $[-\pi, \pi]$; if such an $A$ exists, then any other bounded solution differs from $A$ by a solution $Q$ of $\exp (i Q)=I$ (the identity). In the particular case where $V$ is a unitary operator in Hilbert space, such a solution $A$ exists and is self-adjoint; this is still true if the restriction on the eigenvalues of $V$ is removed, and the uniqueness of $A$ results then from the additional condition that $-\pi$ not be an eigenvalue of $A$. Any other (possibly unbounded) self-adjoint solution again differs from $A$ by a solution $Q$ of $\exp (i Q)=I$. The explicit forms of the solutions $Q$ are studied in both cases; in particular, these are characterized by the existence of a family of operators $P_{n}$ such that $Q=2 \pi \sum_{n-\infty}^{\infty} n P_{n}, \sum_{n=-\infty}^{\infty} P_{n}=I$, and $P_{i} P_{j}=0$ when $i \neq j$. Example: The infinitesimal generator of the Titchmarsh semi-group $T_{\alpha}$ (see Bull. Amer. Math. Soc. Abstract 60-5-611) yields, for a certain class of Banach spaces, a solution $A$ of $\exp (i A)=T_{1}$ having the required uniqueness properties. The proof of Theorem $I$ is based on the integral representation of $V$ developed by Lorch [Trans. Amer. Math. Soc. vol. 49 (1941) pp. 18-40]. (Received July 6, 1954.)

709. R. B. Leipnik: Interchange theorems for limits of iterated integrals.

Let $\Phi=\left\{\Phi_{m, n}\right\}$ be a double sequence of functions integrable with respect to $\mu \times \nu$ measure, and let $L_{1}, L_{2}, L_{3}, L_{4}$ denote limit w.r.t. $m$, limit w.r.t. $n$, integration w.r.t. $\mu$, and integration w.r.t. $\nu$, respectively. There are $4 !=24$ possible orders in which $L_{1}, L_{2}, L_{3}$, and $L_{4}$ can be applied to $\Phi$. Conditions are derived for interchange of order. 
Applications are made to interchange theorems for improper double integrals of the Fourier type. (Received July 16, 1954.)

710. H. A. Linstone: Singular perturbations of linear differential equations in the complex plane.

For large values of the parameter $\lambda$, the asymptotic properties of the equation $y^{(n)}+\sum_{j=1}^{n} \alpha_{j}(x) y^{(n-j)}+\lambda^{n-m}\left[x^{p} \widetilde{\beta}_{0}(x) y^{(m)}+\sum_{j=1}^{m} \beta_{j}(x) y^{(m-j)}\right]=0, n>m$, with $n, m$, and $p$ positive integers and $\widetilde{\beta}_{0}(0) \neq 0$, are studied. The independent variable $x$ ranges over a bounded region $S$ of the complex plane, in which, for all $j$, the coefficients $\alpha_{j}(x)$ and $\beta_{j}(x)$ are analytic, and which contains no zeros of $x^{p} \widetilde{\beta}_{0}(x)$. No loss of generality results if $\widetilde{\beta}_{0}(x) \equiv 1$ and arg $\lambda=0$ are assumed. The use of asymptotic expansions in $\lambda^{-1}$ to approximate the solutions involves the Stokes phenomenon. Using the techniques of Birkhoff, Turrittin, and Wasow, regions of validity, corresponding to any location of $x$ in $S$, are found for the $n-m$ dominant-recessive asymptotic approximations and the $m$ balanced asymptotic series. An analysis of an equation of the type $y^{(4)}+\lambda^{2}\left(x^{2} y^{\prime \prime}+r y\right)=0, r=$ constant, by the Laplace contour integral method shows that solutions which are dominant-recessive in the sector obtained by the general theory are balanced in the complementary region, and solutions with balanced behavior in the sector derived previously are dominant in the remaining region. Thus, as for the known cases with coefficients linear in $x$ (e.g., Wasow, Ann. of Math. vol. 52 (1950) pp. 350-361), the minimum sectors of validity predicted by the general theory define maximum regions. (Received July $6,1954$. )

711. A. J. Lohwater (p) and George Piranian: Sets of radial discontinuity of bounded analytic functions.

Let $E$ be a set of points on the unit circle, of measure zero and type $F_{\sigma}$ (or of type $G_{\delta \sigma}$ and subject to certain metric conditions which cannot be described here); then there exists a function $f(z)$, regular and bounded in $|z|<1$, such that $\lim _{r \rightarrow 1} f\left(r e^{i \theta}\right)$ exists if and only if the point $e^{i \theta}$ does not lie in $E$. If the set $E$ above is closed, there exists a function $f(z)$, regular and bounded in $|z|<1$, and with the following properties: On each radius $O P$ which terminates in $E, \lim$ sup $|f|=1$ and $\lim \inf |f|=0$; on every other radius of the unit disc, the radial limit of $f$ exists and has modulus 1 , except for a denumerable set of radii on which $f \rightarrow 0$. (Received July 19, 1954.)

712t. L. L. Philipson: The asymptotic character of the solutions of a class of ordinary linear differential equations depending on a parameter.

The asymptotic behavior for large values of a parameter $\lambda$ of the solutions of $\sum_{k=0}^{n} a_{k} y^{(n-k)}+\lambda \sum_{k=0}^{m}\left(b_{k}+c_{k} x\right) y^{(m-k)}=0$ is studied in detail so as to illuminate the Stokes variations in the asymptotic representations about the transition point $-b_{0} / c_{0}$ in the complex $x$-plane. The $a$ 's, $b$ 's, $c^{\prime}$ 's are constants with $c_{0} \neq 0 ; n>m>0$. Employing the Laplace contour integral, it is found that if $p=n-m \geqq 2$, there exist $p+1$ solutions each of which tends, as $\lambda \rightarrow \infty$, exponentially to infinity or zero when $x$ is not on a certain ray from the transition point, but when $p=1$, at least in certain cases, an entire sector of width $\pi / 2$ is excluded. In the interior of the excluded sector the solution tends to a finite function of $x$. There also exist in general solutions which converge, as $\lambda \rightarrow \infty$, to functions of $x$ either in complete or partial sectors about the transition point. In the latter circumstance, the solution diverges exponentially 
in the interior of the remaining sector, of width $p \pi /(p+1)$. Expressions for the solutions' "mixed" behavior on the bounding rays of the various sectors are established also. It is proved that a fundamental set of solutions of the above types generally exists. (Received July 6, 1954.)

\section{D. H. Potts: Elementary integrals.}

It is shown that by accepting a restricted concept of elementary function one is able to simplify the treatment of problems on elementary integrals as presented in Ritt, Integration in finite terms. The restriction is to narrow the usual idea of "algebraic operation" to one involving only rational operations and the extraction of root. This enables the author to prove a form of the Liouville-Ostrowski theorem without recourse to techniques as advanced as those used in Ritt, e.g. complex variable theory. (Received July 16, 1954.)

714. O. W. Rechard: Invariant measures for many-one transformations.

Let $X$ be an abstract space and $m$ a finite measure defined on a completely additive class $\mathfrak{X}$ of measurable subsets of $X$. Let $T$ be a transformation (not necessarily oneone) of $X$ onto itself such that the complete inverse image of every measurable set is measurable and $m\left[T^{-1}(A)\right]=0$ if $m(A)=0$. Then the following theorem is proved: There exists a finite measure $m^{*}$ defined on $\mathfrak{X}$, absolutely continuous with respect to $m$, and invariant with respect to $T$ (i.e. $m^{*}\left[T^{-1}(A)\right]=m^{*}(A)$ ) if and only if the set functions $m_{n}(A)=m\left[T^{-n}(A)\right]$ are uniformly absolutely continuous with respect to $m$. The measure $m^{*}$ can be represented as the indefinite integral of a summable function $m^{*}(A)=\int_{A} f d m$. If $P$ is the set of points for which $f$ is positive, then, to within sets of $m$-measure zero, $P \subset T^{-1}(P) \subset T^{-2}(P) \subset \cdots$ and $X=\bigcup_{n=0}^{\infty} T^{-n}(P)$. This theorem is, in a sense, an extension of a theorem on one-one transformations due to M. Cotlar and R. A. Ricabarra (Sobre un Teorema de E. Hopf, Revista Union Mat. Argentina vol. 14 (1949) pp. 49-63). However, the method of proof is entirely different from that used by the above authors. Invariant measures for special transformations of the unit interval are studied. (Received July 15, 1954.)

\section{P. V. Reichelderfer: On the behavior of essential continua in the product of two transformations.}

Given bounded domains $D^{\prime}$ and $D^{\prime \prime}$ in euclidean $n$-space let $T^{\prime}$ be a continuous transformation from $D^{\prime}$ into $D^{\prime \prime}$ and let $T^{\prime \prime}$ be a continuous transformation from $D^{\prime \prime}$ into a bounded portion of $n$-space. If $C^{\prime}$ is an essential maximal model continuum for $T^{\prime}$ and $T^{\prime} C^{\prime}$ is an essential maximal model continuum for $T^{\prime \prime}$ (see T. Rado and P. V. Reichelderfer, On n-dimensional concepts of bounded variation, absolute continuity and generalized jacobian, Proc. Nat. Acad. Sci. U.S.A. vol. 35 (1949) pp. 678-681) it is shown that $C^{\prime}$ is also an essential maximal model continuum for $T^{\prime \prime} T^{\prime}$. Moreover, if $C^{\prime}$ is essentially isolated for $T^{\prime}$ and $T^{\prime} C^{\prime}$ is essentially isolated for $T^{\prime \prime}$, then $C^{\prime}$ is essentially isolated for $T^{\prime \prime} T^{\prime}$ and the product law holds for their essential local indices. Conversely, if $C^{\prime}$ is an essentially isolated essential maximal model continuum for $T^{\prime \prime} T^{\prime}$, then $C^{\prime}$ is an essentially isolated essential maximal model continuum for $T^{\prime}$ and $T^{\prime} C^{\prime}$ is an essentially isolated maximal model continuum for $T^{\prime \prime}$. (Received July 15,1954 .)

716t. Walter Rudin: Images of radii under analytic mappings of the unit circle. 
Given a sequence $\left\{a_{k}\right\}$ of positive numbers such that $\sum a_{k}^{2}$ converges but $\sum a_{k}$ diverges, it is shown that there exists a sequence of positive integers $n_{k}$, tending to infinity sufficiently rapidly, such that the function $f(z)=\sum a_{k} z^{n} k$ maps every radius of the unit circle into a non-rectifiable curve, although the power series converges at almost all points of the circumference, and although $f$ is of class $H_{p}$ for every $p<\infty$. Furthermore, $f$ is the quotient of two bounded analytic functions $g$ and $h$, and it is shown that there exists a complex number $c$ such that the bounded function $g+c h$ maps almost all radii into non-rectifiable curves. The exceptional set of radii is also of the first category. (Received June 10, 1954.)

\section{7t. Walter Rudin: On mapping properties of Blaschke products.}

If $f$ is analytic in the unit circle, let $V(f ; \theta)=\int_{0}^{1}\left|f^{\prime}\left(r e^{i \theta}\right)\right| d r ;$ i.e. $V(f ; \theta)$ is the length of the curve into which $f$ maps the radius which terminates at the point $e^{i \theta}$. It is shown that there exists a Blaschke product $B$ such that $V(B ; \theta)=\infty$ for almost all $\theta$. On the other hand, if the zeros $z_{n}$ of $B(z)$ satisfy the condition $\sum\left(1-\left|z_{n}\right|\right)\left|\log \left(1-\left|z_{n}\right|\right)\right|$ $<\infty$, instead of merely $\sum\left(1-\left|z_{n}\right|\right)<\infty$, then $\int_{0}^{2 \pi} V(B ; \theta) d \theta<\infty$, so that, in particular, almost all radii are mapped into rectifiable arcs. (Received June 14, 1954.)

\section{Walter Rudin: Radial cluster sets of analytic functions.}

Let $E$ be a set of the first category on the circumference $C$ of the open unit disc $U$; the linear measure of $E$ may be $2 \pi$. Theorem I: For every complex-valued function $F$, continuous in $U$, there is a function $f$, analytic in $U$, such that $\lim _{r \rightarrow 1}\{f(r x)-F(r x)\}$ $=0$ for every $x \in E(0<r<1)$. The proof is based on Mergelyan's theorem on approximation of continuous functions by polynomials. A consequence is Theorem II: Given any continuum $K$ on the Riemann sphere, there exists a function $f$, analytic in $U$, whose radial cluster set at every point of $E$ is precisely $K$. In particular, there exists (1) a harmonic function $u$ in $U$ which has finite radial limits at every point of $E$ and whose conjugate $v$ has no radial limit at any point of $E$, (2) an analytic function $f$ in $U$ such that $\lim _{r \rightarrow 1}|f(r x)|=1$ for every $x \in E$, whereas $\lim _{r \rightarrow 1} f(r x)$ exists for no $x \in E$, (3) an analytic function $f$ in $U$ such that $\lim _{r \rightarrow 1} f(r x)=0$ and $\lim _{r \rightarrow 1} \arg f(r x)=0$ for every $x \in E$. (Received July 6, 1954.)

\section{A. L. Shields: Exceptional sets for uniform distribution.}

The following theorem is proven: Let $a_{1}<a_{2}<\cdots$ be positive integers such that inf $\left(n / a_{n}\right)>0$. Let $E$ be the set of points $x \in[0,1]$ such that the fractional parts $\left(a_{n} x\right)$ are not uniformly distributed. Then, Hausdorff dimension of $E$ is $\leqq 2(1-\alpha)$. This extends a theorem of I. I. Shapiro-Piatetsky, Mat. Sbornik vol. 30 (1952) pp. 669-676. (Received July 19, 1954.)

\section{0t. I. M. Singer and John Wermer: Derivations of commutative Banach algebras.}

A derivation on an algebra is a linear transformation $D$ such that $D(a b)=D(a) b$ $+a D(b)$ for $a$ and $b$ in the algebra. Kaplansky conjectured that the only derivation on a semisimple commutative Banach algebra was the 0 derivation. It is proved that if $D$ is a continuous derivation on an arbitrary commutative Banach algebra, then the range of $D$ is contained in the radical of the algebra. Thus the conjecture is true if $D$ is continuous. If $b$ is an element of any Banach algebra, bracket by $b$ is a continuous derivation and hence the theorem cited above has as corollaries several facts about commuting brackets. (Received July 19, 1954.) 


\section{Vikramadity Singh and W. J. Thron (p): A family of best twin convergence regions for continued fractions.}

Combining methods of the theory of linear fractional transformations and their iteration with an application of the Stieltjes-Vitali theorem it has been proved that the continued fraction $1+k\left(c_{n}^{2} / 1\right)$ converges if for all $n \geqq 1,\left|c_{2 n-1}\right| \leqq \rho$ and $\left|c_{2 n} \pm i\right| \geqq \rho$, where $0<\rho<1$. This result gives a set of best twin convergence regions and is an improvement of an earlier theorem by one of the authors (Duke Math. J. vol. 10 (1943) pp. 667-685). (Received July 6, 1954.)

\section{K. T. Smith: A generalization of an inequality of Hardy-Little-} wood and applications.

In this paper a generalization of a well-known inequality of Hardy and Littlewood is given. Consider a measure $\mu$ on a metric space $B$ and make the hypotheses: (a) each closed sphere $S(x, r)$ is measurable and has finite measure; (b) there is a constant $k$ such that for each $x$ and $r, \mu[S(x, 4 r)] \leqq k \mu[S(x, r)]$; (c) if $\left\{S_{n}\right\}$ is a sequence of closed spheres such that $\mu\left(S_{n}\right) \rightarrow 0$, then the diameters $\delta\left(S_{n}\right) \rightarrow 0$; (d) if $\left\{S_{n}\right\}$ is a sequence of closed spheres such that $\delta\left(S_{n}\right) \rightarrow \infty$, then $\mu\left(S_{n}\right) \rightarrow \infty$. The inequality obtained asserts that if $p>1$, then $\int_{B} \bar{f}(x)^{p} d \mu \leqq A_{p} \int_{B}|f(x)| p d \mu$, when $\bar{f}(x)$ is defined as the supremum of the averages of $|f(x)|$ over the closed spheres centered at $x$. The applications given concern the case where the metric space $B$ is a smooth surface (bounded curvature suffices) bounding a domain $D$ in $n$-dimensional Euclidean space and $\mu$ is the area measure on $B$. The results are of the following type. Let $f(x)$ be the boundary function of a function $f(P)$ harmonic in $D$. For each $x$ on $B$ let $\hat{f}(x)$ be the upper bound of the values of $|f(P)|$ along the normal to $B$ through $x$. Then for each $p>1$, there is a constant $A_{p}$ such that $\int_{B} \hat{f}(x)^{p} d \mu \leqq A_{p} \int_{B}|f(x)|{ }^{p} d \mu$. (The case where $D$ is the unit circle in the plane was treated by Hardy and Littlewood.) There are given also some applications to subharmonic functions. (Received July 19, 1954.)

723. Andrew Sobczyk: Sections and projections of convex bodies. Preliminary report.

If $\alpha_{1}, \cdots, \alpha_{n}$ are basis vectors for real linear space $E_{n}$, define $n$ projections $P_{j}$ by $P_{j}\left(x_{1} \alpha_{1}+\cdots+x_{j} \alpha_{j}+\cdots+x_{n} \alpha_{n}\right)-x_{j} \alpha_{j}, j=1, \cdots, n$. Denote by $L_{j}$ the line consisting of the points $\left\{c \alpha_{j}\right\}$ for all real $c$. A convex region $K$ in $E_{n}$ is of standard type in case there exist a translate $C=K+w$, and a choice of basis $\left\{\alpha_{j}\right\}$, such that each projection $P_{j} C$ coincides with the section $L_{j} \cap C, j=1, \cdots, n$. This paper partially answers, affirmatively, the following question. Is every convex region $K$ in $E_{n}$ of standard type? In other words, does every $K$ have a set of $n$ one-dimensional sections, such that $K$ is intermediate between the convex hull and the Cartesian product of the sections? (Received July 15, 1954.)

\section{E. W. Titt: An alternative method of evaluating Hadamard's} finite part.

This paper presents an alternative to Hadamard's Taylor expansion method of evaluating the finite part of a divergent integral. In this paper integration by parts does the work which is accomplished by the Taylor expansion in Hadamard's method. On the other hand the integration by parts technique is more flexible since here neither factor need be expressed as a power function. The equivalence of the two methods is established for the case of single integrals. The new technique is illustrated with the 
solution of an ordinary equation with a nonintegrable forcing term. The interest in the new method arose out of the problem of reconciling Hadamard's integration formula in terms of finite parts with the type of integration formula to be found in McCulley and Titt (Journal of Rational Mechanics and Analysis vol. 2 (1953) pp. 443-484). (Received July 19, 1954.)

725. F. M. Wright: On the backward extension of positive definite Hamburger moment sequences.

Let $\left\{\mu_{n}\right\}(n=0,1,2, \cdots)$ be a given positive definite Hamburger moment sequence. Using properties of the polynomials $X_{p}(z)$ and $Y_{p}(z)(p=0,1,2, \cdots)$, which are the solutions of a certain second order recurrence system $L_{p}(\eta, z)=0$ $(p=0,1,2, \cdots)$, for certain initial conditions, the author, by using what is believed to be a relatively simple and direct method, obtains and enlarges upon the principal results of Hamburger (Math. Ann. vol. 82 (1921) pp. 168-189) relative to the problem of extending the given Hamburger moment sequence backward. A necessary and sufficient condition for a first backward extension is obtained which is first stated in terms of the polynomials $\left\{X_{p}(z)\right\}$ and $\left\{Y_{p}(z)\right\}(p=0,1,2, \cdots)$, and then in terms of the given moments. The necessary and sufficient condition for an indeterminate Hamburger moment sequence in terms of the given moments due to Hamburger and reproved by M. Riesz (Arkiv för Mathematik, Astronomi och Fysik vol. 16 (1921) article no. 12) is also proved in this paper in a relatively simple manner. (Received July 9, 1954.)

\section{6t. L. C. Young: Analogues of an inequality due to Aronszajn and Choquet. Preliminary report.}

The area of a surface of finite topological type is not less than the product $K L L^{\prime}$ where $K$ is an absolute constant and where $L, L^{\prime}$ are lengths of two curves on the surface. One curve can be taken as any 1-cycle of minimal length in its homology class, the other as a 1-cycle of minimal length not homologous to zero. There is a similar result in terms of homotopy. (Received July 19, 1954.)

\section{L. C. Young: A variational algorithm.}

Parametric surface integrands and curvilinear integrands are not restricted to be continuous. An integrand is exact if, on every closed surface, or on every closed curve, its integral vanishes. Two variational problems with the same boundary conditions are termed equivalent if the difference of their integrands is exact; curves and surfaces are understood to be generalized and the notion of boundary is that of Fleming and Young (Bull. Amer. Math. Soc. Abstract 58-6-615). With these conventions, it is shown that the principle of minimum in a variational problem with given boundary is tantamount to the existence of an equivalent problem with a non-negative integrand which vanishes on some admissible surface, or curve. (Received July 19, 1954.)

\section{8t. L. C. Young: On generalized surfaces of finite topological type.}

A Dirichlet surface of a given topological type is one for which the boundary curve describes a given finite set of simple closed curves $C_{i}$ together with pairs of Fréchet equivalent arcs with the appropriate characteristic. A generalized surface of finite type is the limit (in the sense of generalized surfaces) of Dirichlet surfaces of bounded topological type and given $C_{i}$. It is shown that the track of a generalized surface of finite type is a Dirichlet surface of the same type and a general representa- 
tion theorem is obtained which is almost completely analogous to the one for generalized curves. In the case in which the generalized surface is the solution of an acceptable variational problem its track is shown to be continuous. If the problem has a convex integrand, its solution coincides with this track. A corollary of these results is the extension to surfaces of given topological type of a recent existence theorem due to Sigalov, Danskin and Cesari. (Received July 19, 1954.)

\section{Applied Mathematics}

729. W. L. Bade and Herbert Jehle (p): Specific interactions between identical macromolecules.

A fundamental biophysical problem, originally stated by the geneticist $\mathrm{H}$. J. Muller, is to explain why nucleic acids and proteins "specifically" attract like molecules, discriminating for an exactly identical partner. The molecules in question present a periodic (presumably helical) structure of great rigidity. They are composed of a backbone of repeating units with a side group attached to each unit. These macromolecules should be represented by an orchestra of electric dipole oscillators vibrating because of the temperature motion. The authors are investigating those vibrations to find out which types of quantum mechanical models of pairs of macromolecules exhibit a specific Van der Waals attraction. Such attraction has been conjectured on the basis of arguments in terms of Bohr's correspondence principle (Proc. Nat. Acad. Sci. U. S. A. vol. 36 (1950) p. 238). Specificity of attraction is analyzed by a study of various "detunings" of one of the macromolecules (slight changes of the identity) of the pair. That analysis was greatly advanced by a complete investigation of a model in which each molecule is represented by only two oscillators (W. L. Bade). It has substantiated the implications of the earlier correspondence arguments for this $2+2$ oscillator model. The analysis of an $N+N$ oscillator model of the macromolecule pair (J. M. Yos) is preceded by a group theoretical analysis of vibrations of identical macromolecule pairs and of their detunings. (Research supported by the Research Corporation and National Science Foundation.) (Received July 19, 1954.)

730. R. G. Blake: A problem in laminated orthotropic material under plane strain.

Prager and Synge [Quarterly of Applied Mathematics vol. 5 (1947) pp. 241-269] have developed a method of approximation in elasticity based on the concept of function space. This method is used to obtain a first approximation to a problem of plane strain in a rectangle consisting of three layers of orthotropic material cemented together with the two outer layers similar. The line of separation of the layers is taken as $y= \pm c$ and the boundaries of the rectangle as $x= \pm w c, y= \pm t c$. The boundary conditions to be satisfied are: at $y=t c, \tau_{y y}=K x^{2 b}, \tau_{x y}=0$; at $y=-t c, \tau_{y y}=\tau_{x y}=0$; at $x= \pm w c, \int_{-t c}^{t c} \tau_{x y} d y=\mp K(w c)^{a+1} /(a+1), \int_{-t c}^{t c} \tau_{x x} d y=\int_{-t c}^{t o} \tau_{x x} y d y=0$. (Received July 19, 1954.)

\section{R. V. Churchill: A generalized Fourier integral formula.}

The solution of certain types of boundary value problems in partial differential equations, including problems of some importance in applied mathematics, depends on a representation of a function $F(x)$ on the semi-infinite interval $x>0$ in terms of the characteristic functions $y(x, \lambda)$ of the problem $y^{\prime \prime}+\lambda^{2} y=0, m y^{\prime \prime}(0, \lambda)-k y^{\prime}(0, \lambda)$ $+h y(0, \lambda)=0, y(x, \lambda)$ bounded for $x \geqq 0$. Here $m, k$, and $h$ are nonnegative constants. Since $y^{\prime \prime}(0, \lambda)=-\lambda^{2} y(0, \lambda)$ the boundary condition on $y$ involves the parameter $\lambda$ 
explicitly if $m \neq 0$. The characteristic functions are $y(x, \lambda)=\cos [\lambda x-\alpha(\lambda)]$, where $\tan \alpha(\lambda)=\left(h-m \lambda^{2}\right) /(k \lambda),-\pi / 2 \leqq \alpha(\lambda) \leqq \pi / 2$ and $\lambda$ is real and nonnegative. When $F(x)$ satisfies conditions under which it is represented by its Fourier sine and cosine integral formulas, the generalization $\pi F(x)=2 \int_{0}^{\infty} y(x, \lambda) d \lambda \int_{0}^{\infty} F(\mu) y(\mu, \lambda) d \mu$ of those formulas is established for $x>0$ with the aid of the Riemann-Lebesgue theorem. This formula includes as a special case $(m=0)$ the generalization arrived at formally by W. Karush [Journal of Applied Physics vol. 23 (1952) pp. 492-494] and by the author [Modern operational mathematics, 1944, p. 210]. (Received July 19, 1954.)

732. Jim Douglas, Jr.: On the integration of $U_{x x}+U_{y y}=U_{t}$ by implicit methods.

Three implicit difference equation analogues of the heat flow equation in two space variables are studied. Under proper hypotheses the solution of each is shown to converge to the solution $u(x, y, t)$ of the differential equation for any ratio of $\Delta t$ to $(\Delta x)^{2}$ $=(\Delta y)^{2}$ as $\Delta t \rightarrow 0$ with the error in each case being $\mathrm{O}(\Delta t)$. Let $f_{i, j, n}=f(i \Delta x, j \Delta y, n \Delta t)$ and $\Delta_{x}^{2} f_{i, j, n}=\left(f_{i+1, j, n}-2 f_{i, j, n}+f_{i-1, j, n}\right) /(\Delta x)^{2}$. Then, let $W_{i, j, 0}=U_{i, j, 0}$ and $W_{i, j, n}$ $=U_{i, j, n}$ on the boundary of the unit square for $n>0$. The three difference equations are (I) $\Delta_{x}^{2} W_{i, j, n+1}+\Delta_{y}^{2} W_{i, j, n+1}=\left(W_{i, j, n+1}-W_{i, j, n}\right) / \Delta t$; (II) $\Delta_{x}^{2} W_{i, j, n+1}+\Delta_{y}^{2} W_{i, j, n+1}$ $+\Delta_{x}^{2} W_{i, j, n}+\Delta_{y}^{2} W_{i, j, n}=2\left(W_{i, j, n+1}-W_{i, j, n}\right) / \Delta t ; \quad$ and (III) $\Delta_{x}^{2} W_{i, j, 2 n+1}+\Delta_{y}^{2} W_{i, j, 2 n}$ $=\left(W_{i, j, 2 n+1}-W_{i, j, 2 n}\right) / \Delta t ; \Delta_{x}^{2} W_{i, j, 2 n+1}+\Delta_{y}^{2} W_{i, j, 2 n+2}=\left(W_{i, j, 2 n+2}-W_{i, j, 2 n+1}\right) / \Delta t$. Equation (III), while appearing the most formidable, is in fact much the easiest to evaluate as the linear equation can be solved by well known methods in $9(\Delta x)^{-2}$ calculations per time step. Practical aspects of the methods and applications to Laplace's equation are given in abstract by Peaceman and Rachford (Abstract 735). (Received July 19, 1954.)

\section{T. C. Doyle: Invariantive analytic representation of a finite} deformation of a continuous medium.

The material (undeformed) points are referred alternatively to (a) an absolute (fixed) orthonormal triad, (b) a moving orthonormal material triad, (c) a moving curvilinear material triad, and similarly for the deformed (spatial) points. Any vector may be given by specifying its components in any one of these five reference triads and transformations between these sets of components are accomplished by given matrices of direction cosines and displacement gradients. There exist semi-intrinsic parameterizations (convected coordinates) in which the curvilinear strain tensor components coincide with the metric tensor components in the semi-intrinsic base. (Received June 24, 1954.)

\section{4t. L. E. Payne: Inequalities for certain eigenvalues of a membrane.}

Let $D$ be a closed domain with boundary $C$ in the $x y$ plane and let $u(x, y)$ satisfy the differential equation $\Delta u+k u=0$ in $D$ (where $k$ is a positive constant) and either (1) $u=0$ on $C$ or (2) $\partial u / \partial n=0$ on $C$. Denote the eigenvalues in case (1) by $\lambda_{1} \leqq \lambda_{2} \leqq \lambda_{3}$ $\leqq \cdots$, and in case (2) by $0=\mu_{1} \leqq \mu_{2} \leqq \mu_{3} \leqq \cdots$. It has been shown by Szegö (Journal of Rational Mechanics and Analysis vol. 3 (1954) p. 354) that $\mu_{2} \leqq p \lambda_{1}$ where $p$ is the constant giving equality if $D$ is a circle. In this article for a convex domain $D$ the following inequalities are obtained: (3) $\mu_{2}<\lambda_{1}-2 /(\rho h)_{\max }$ and (4) $\mu_{3}<\lambda_{1}-\left(2^{1 / 2}-1\right) /(\rho h)_{\max }$ $\leqq \lambda_{1}$, where $\rho$ is the radius of curvature of $C$, and the value of $h$ at any point $P$ on $C$ is the distance from an arbitrary origin inside $D$ to the line tangent to $C$ through $P$. This research was supported by the Office of Scientific Research, U.S.A.F. (Received June 9, 1954.) 
735t. D. W. Peaceman and H. H. Rachford, Jr.: On the numerical integration of two-dimensional parabolic and elliptic partial differential equations.

I. Stepwise solution of $U_{x x}+U_{y y}=U_{t}$ through explicit difference equations requires an excessive number of steps owing to stability limitations. Implicit formulations similar to those used in the one-dimensional analogue have been considered. Approximation of the $x$ - and $y$-derivatives in terms of the unknown values at the end of the step yields a stable solution for all $\Delta t$ but requires at each step solution of $N^{2}$ $=(\Delta x)^{-2}$ simultaneous equations, best solved by iterative methods which converge more slowly as $\Delta t$ increases. A non-iterative implict scheme, stable for all $\Delta t$, is achieved by a two-step formulation wherein the $x$ - or $y$-derivative is alternately expressed in terms of the unknown values. At each step $N$ sets of $N$ simultaneous equations are solved directly, requiring $9 N^{2}$ arithmetic operations. Proof of convergence is given in a paper by Douglas (Abstract 732). II. This alternating direction implicit formulation may be used to solve the elliptic equation $U_{x x}+U_{y y}=0$ by iteration. A trial solution is carried through a succession of "time steps" wherein $\Delta t$ becomes an iteration parameter. A set of $\Delta t^{\prime}$ 's which optimizes convergence is readily computed. The work to reduce the error by $10^{-3}$ for large $N$ is $34 N^{2} \log N$ operations. This is compared with $15 N^{3}$ for optimum extrapolated Liebmann iteration procedures. (Received July 19, 1954.)

\section{M. H. Protter: On some problems in transonic flow.}

Existence and uniqueness theorems are established for certain boundary value problems for the equation $K(y) u_{x x}+u_{y y}=0$ where $K(y)$ is monotone increasing, $K(0)=0$. These problems occur in the two-dimensional transonic flow of an ideal gas between two plane converging walls. The uniqueness result is more general than previous ones obtained in that it contains no restrictions on the domain in the case where $K(y)$ is the particular function occurring in the gas dynamical case. The existence follows from a combination of the uniqueness and a previous result of the author. (Received July 8, 1954.)

\section{7t. Domina E. Spencer: The ordinary differential equations of mathematical physics.}

An important step in the solution of the partial differential equations of field theory is the classification and solution of the ordinary differential equations obtained by separation of variables. A convenient method of classification of these ordinary differential equations stems from the Fuchsian theory, as has been demonstrated by Klein and Bôcher. The present paper extends previous investigations by considering equations obtained by separation of the Laplace and Helmholtz equations in the eleven coordinate systems of Eisenhart and the eleven symmetric cyclidal systems of Bocher. These twenty-two coordinate systems comprise all the separable systems that are likely to be used in practice. Despite the large number of separable coordinate systems, each of which has three separation equations, there are only twelve distinct differential equations, and four of these are solved by elementary functions. The differential equations are classified with respect to their singularities, and their solutions are indicated. (Received July 19, 1954.)

738. H. F. Weinberger: $A$ Rayleigh-Ritz procedure for unbounded perturbations. 
Let $A$ be a positive symmetric differential operator with discrete spectrum in an incomplete Hilbert space (functions satisfying differentiability and boundary conditions). Let its eigenvalues $\mu_{n}$ and eigenfunctions $v_{n}$ be known. Let $C$ be another differential operator which satisfies, for all unit vectors $w,(C w, C w)^{1 / 2} \leqq \phi((A w, w))$ where $\phi(x)$ is a function satisfying (1) $0<\phi^{\prime}(x)<1$; (2) $\lim \sup _{x \rightarrow \infty} \phi(x) / x \leqq 0.39$; and (3) the quantity $\left[\phi \phi^{\prime} /\left(1-\phi^{\prime}\right)\right]^{\prime}<0$ for sufficiently small $x$ and changes sign at most once. Then one can calculate the maximum error made in approximating any particular eigenvalue of the perturbed operator $A+C$ with the same boundary conditions by means of the Rayleigh-Ritz upper bound obtained by using the vectors $\left(v_{1}, v_{2}, \cdots, v_{N}\right)$. This error bound is given in the terms of the known quantities $\mu_{n}$, and can be made arbitrarily small by choosing $N$ sufficiently large. The conditions (1), (2), and (3) are satisfied for $\phi=c x^{\alpha}$ with $\alpha<1$, so that if $A$ is an elliptic differential operator, $C$ may be any operator of lower order. By a slight modification the method can be applied even when the eigenvalues of $A+C$ are to be found with respect to a new norm which is bounded above and below with respect to the original norm. Application is made to the natural frequencies of a vibrating clamped plate. This research was supported by the United States Air Force through the Office of Scientific Research. (Received July 19, 1954.)

739. J. M. Yos: Group theoretical analysis of vibrations of macromolecule pairs.

In the investigation described by $\mathrm{H}$. Jehle and W. L. Bade it is necessary to expand a symmetric function of the characteristic roots of the potential energy matrix of the molecule pair in terms of small "detunings" of the original undetuned pair. This expansion is facilitated by considering the symmetry group of the undetuned system. The transformations of this group produce a representation of the group in the space of detunings, and if the detuning parameters are chosen to reduce this representation the expansion takes on its simplest form. The argument shows that certain coefficients in the expansion vanish identically, and that others are necessarily equal. (Research supported by the National Science Foundation.) (Received July 19,1954.)

\section{GeOMETry}

\section{0t. M. W. Al-Dhahir: Configurational characterizations of com-} mutativity. I.

Let $S_{n}$, with $n$ odd, be a projective $n$-space defined over a division ring $\mathcal{F}$. The fundamental theorem on plane complete quadrangles (Veblen and Young, Projective geometry, p. 101) is generalized to $n$ dimensions in the following form: Let $012 \cdots n$ and $0^{\prime} 1^{\prime} 2^{\prime} \cdots n^{\prime}$ be two complete hyper-plane $(n+1)$-points. If all, but one, of the $n(n+1) / 2$ lines of one meet the correspondingly "opposite faces" of the other, then the remaining line of the first meets its corresponding face of the second if, and only if, $\mathcal{F}$ is a field. Through an $n$-dimensional projection, a "similar" result is obtained in even spaces. Thus, a configurational criteria for the commutativity of the space is obtained. This generalization involves the Möbius proposition for $n=3$, and specializes to the Pappus theorem for $n=2$, and, as such, it may be considered as an extension of both of these propositions also. (Received June 21, 1954.)

\section{1t. Rafael Artzy: Self-dual configurations and their Levi graphs.}

In the Levi graph (Coxeter, Bull. Amer. Math. Soc. vol. 56 (1950) pp. 413-455) of a self-dual configuration there is a one-to-one correspondence between all point- 
nodes and all line-nodes. Each two corresponding nodes are abstractly identified without altering the joining branches. The reduced Levi graph (RLG) thus generated has only half the number of nodes, but it may have loops representing points incident with dually corresponding lines in the configuration. A configuration may have several nonisomorphic RLG's, but no RLG represents more than one configuration. Self-dual specializations of a configuration imply in the RLG certain modifications which are studied. The RLG's of special configurations are dealt with. Thus the RLG of Desargues' configuration is Petersen's graph; several self-dual specializations yield Petersen's graph with loops added. The method is useful for the study of finite geometries: the RLG's of PG $(2,3)$ and PG $(3,2)$ contain the RLG's of several well known configurations as subgraphs, thus implying the possibility of constructing these configurations in the respective geometry. (Received July 12,1954.)

742. P. O. Bell: On projective tensors of a surface $S$ with respect to a surface $S^{\prime}$.

Let $g_{\alpha \beta}$ and $d_{\alpha \beta}$ denote the first and second fundamental projective tensors of a surface $S\left(u^{1}, u^{2}\right)$ with respect to a surface $S^{\prime}\left(u^{1}, u^{2}\right)$ and let $R_{\alpha \beta}$ and $K$ denote the projective curvature tensor and projective total curvature of $S$ relative to $S^{\prime}$, respectively. ( $g_{\alpha \beta}$ and $K$ were introduced in Trans. Amer. Math. Soc. vol. 60 (1946) pp. 34-39. $R_{\alpha \beta}$ and $d_{\alpha \beta}$ were introduced in Duke Math. J. vol. 18 (1951) pp. 697-705.) Infinitely many pairs of surfaces $\left(S^{\prime}, S^{\prime \prime}\right)$ exist such that the tensor $R_{\alpha \beta}$ of $S$ relative to $S^{\prime}$ and the tensor $K g_{\alpha \beta}$ of $S$ relative to $S^{\prime \prime}$ are related by the equation $R_{\alpha \beta}+K g_{\alpha \beta} \equiv 0$. Corresponding points $x, x^{\prime}, x^{\prime \prime}$ of such surfaces $S, S^{\prime}, S^{\prime \prime}$ are collinear. The harmonic conjugate of $x$ with respect to $x^{\prime}$ and $x^{\prime \prime}$ is a point $\sigma\left(u^{1}, u^{2}\right)$ which generates the $R$-associate surface $\Sigma$ of $S$. (See the first paper cited above, p. 38.) A null line of the first fundamental form of $S$ corresponds to a locus of $\sigma$ whose tangent at $\sigma$ intersects the tangent to the null line. A null line $\Gamma_{1}\left(\Gamma_{2}\right)$ of the second fundamental form of $S$ (i.e., an asymptotic curve of $S$ ) corresponds to a locus of $\sigma$ whose tangent at $\sigma$ intersects the tangent to $\Gamma_{2}\left(\Gamma_{1}\right)$ at $x$. This last property completely characterizes $\Sigma$. (Received July $6,1954$.

\section{3t. John DeCicco: Certain generalizations of central and parallel} fields of force in Euclidean space $E_{n}$.

An $(m-1)$-dimensional central field of force with an $(m-1)$-dimensional flat $L_{m-1}^{0}$ as center, in a certain region of $E_{n}$, is one such that the lines of the force vectors at all points of the region intersect $L_{m-1}^{0}$, and there exist $m$ linearly independent force vectors. The lines of force are all contained in $m$-dimensional flats $L_{m}$ passing through $L_{m-1}^{0}$, and the dynamical trajectories are in $(m+1)$-dimensional flats $L_{m+1}$, all of which contain $L_{m-1}^{0}$. Similarly in an $m$-dimensional parallel field of force with the $m$-dimensional direction $\mu_{m}$, the lines of force lie in $m$-dimensional flats $L_{m}$ and the dynamical trajectories are all contained in $(m+1)$-dimensional flats $L_{m+1}$, all of which are parallel to the direction $\mu_{m}$. For the $(m-1)$-dimensional central fields of force, a law which reduces to that of Kepler's second law of planetary motion when $m=1$ is developed. These are the only positional fields of force for which every dynamical trajectory is contained in some $(m+1)$-dimensional flat $L_{m+1}$, and $m$ is the smallest such integer. (Received July $12,1954$. )

\section{J. W. Gaddum: Distance and angle sums on a sphere.}

The angle between two planes in $E_{n}$ can be expressed as a distance between two points in $S_{n-1,1}$ and the angle at a point formed by $n$ hyperplanes can be treated as 
the "area" of a simplex in $S_{n-1,1}$. With the motivation of seeking bounds on the angle sums in a simplex in $E_{n}$, bounds are found for the sum of the distances between points of a set in $S_{n-1,1}$, and for the content of a spherical simplex in terms of the sum of the angles between its faces. If $A$ is the content of a simplex in $S_{n-1,1}$ and $\sum a_{i j}$ the sum of the angles between its faces, it is proved: for $n$ odd $\sum a_{i j} \geqq\left((n-1)^{2} / 4\right) \pi$ $+\left(\left(n^{2}-1\right) / 2 e_{n}\right) A$, for $n$ even $\sum a_{i j} \geqq(n(n-2) / 4) \pi+\left(n^{2} / 2 e_{n}\right) A$, and $\sum a_{i j}$ $\leqq((n-1)(n-2) / 2) \pi+(2(n-1) \pi / e) A$ for all $n$, where $e_{n}=2 \pi^{n / 2} / \Gamma(n / 2)$. If $p_{1}, \cdots$, $p_{k}$ is a global set in $S_{n-1,1}, \sum p_{i} p_{j} \geqq(K-1) \pi$, and for any set $p_{1}, \cdots, p_{k}$ in $S_{n-1,1}$, $\sum p_{i} p_{j} \leqq\left[k^{2} / 4\right] \pi$. All bounds stated are best possible. The methods are elementary. (Received July 19, 1954.)

\section{Michael Goldberg: Basic rotors in spherical polygons.}

In an earlier paper by the author [Journal of Mathematics and Physics vol. 30 (1952) pp. 235-244], a kinematic method was given for generating a special class of spherical rotors which are convex spherical curves rotatable through all orientations while keeping contact with all the sides of a given regular spherical polygon. This class corresponds to the circular-arc rotors in plane polygons. The present paper exhibits another class of spherical rotors generated by kinematic methods. These correspond to the basic rotors in plane polygons, namely, the convex involutes of the hypocycloids. Their analytic expressions are derived. (Received June 28, 1954.)

\section{P. C. Hammer: Convex bodies in linear spaces.}

Let $C$ be a convex set in a linear space $W$. Let $A_{s}$ be the minimal set covered by the union of translations of all line segments in $C$ with translated centers on the origin $\theta$. Let $C_{s}$ be the set obtained by closing $A_{s}$ on every line through $\theta$. Then $C_{s}$ is the symmetroid of $C$. If $x \in C$, then $2 C_{s}+x \supset C$ and $C_{s}$ is the smallest relatively closed convex set centered on $\theta$ with this property. If the intersection of $C_{s}$ with each line through $\theta$ is a proper finite line segment and if $C$ is closed and contains interior points in the topology with $C_{s}$ as a unit sphere, $C$ is a convex body. From this intrinsic definition of a convex body generalizations of results of Klee, Sobczyk, and the author for finite-dimensional spaces are obtained. Two convex sets with the same symmetroid have equivalent breadths in a generalized sense. (Received July 20,1954.)

747. W. R. Hutcherson (p) and N. A. Childress: Surfaces obtained from involutions generated by homographies of periods three, five, and thirteen.

The image of a planar cyclic involution of period three is represented as a surface [Lucien Godeaux, Étude élémentaire sur l'homographie plane de période trois et sur une surface cubique, Nouvelles Annales de Mathématiques vol. 16 (1916) pp. 49-61] of order three in $S_{3}$. Also, the image of a planar cyclic involution of period five is represented as a surface [W. R. Hutcherson and N. A. Childress, Étude d'une involution cyclique de période cinq, Bulletin de l'Académie royale de Belgique (Classe des Sciences) (6) vol. 40 (1954) pp. 103-108] of order five in $S_{4}$. The image of a planar cyclic involution of period thirteen is represented as a surface of order thirteen in $S_{8}$. The plane is in $(13,1)$ correspondence with this surface. A series of five successive projections is performed on this surface, with the point of projection being on the surface which is being projected each time. A rational surface of order one hundred and sixty nine in $S_{9}$ is shown. This surface is in $(13,1)$ correspondence with the image surface. (Received June 3, 1954.) 
748t. W. R. Hutcherson and J. C. Morelock: Concerning a pattern for perfect points.

Using a certain surface [W. R. Hutcherson, Voisage du cinquieme ordre d'une involution de periode 13, Bulletin Société Royale des Sciences de Liège No. 11 (1952) pp. 483-487; Bull. Amer. Math. Soc. Abstract 59-6-556] invariant under an involution of order $p$ (prime number), it is shown that a simple point $P_{3}$ has perfect points within certain order neighborhoods, depending upon $p$. A table, exhibited for the first six neighborhoods when $p \leqq 47$, is followed by several theorems. The largest prime number found in the cataloging of the first twenty neighborhoods was 14251 . (Received June 1, 1954.)

\section{9t. Herbert Knothe: On polyhedrons in multi-dimensional curved} spaces.

The well known theorem that the area of a triangle bounded by great circles on the unit sphere is equal to the sum of the angles minus $\pi$ can be generalized in spaces of constant curvature of an even number of dimensions. By a simple method of combination a formula is derived expressing the volume of an $(n+1)$-hedron in the $n$ dimensional space by means of the solid angles at the vertices and edges. In the same manner the volume of a polyhedron can be expressed by angles. It is supposed that the volume of a regular curved convex body can be expressed by the $g_{i k}$ of the surface. (Received August 30, 1954.)

\section{T. G. Ostrom: Conics in finite projective planes.}

Baer (Bull. Amer. Math. Soc. vol. 52 (1946)) has shown that a polarity in a finite projective plane with $n+1$ points on a line has $n+1$ absolute points unless $n$ is a square and that, if $n$ is odd, no three absolute points lie on the same line. Assuming $n$ odd and not a square, the author defines a conic as the set of absolute points of a polarity. The author investigates the conditions under which such a conic acts, in certain respects, like the classical conics in a Desarguesian plane. These conditions turn out to be Desargues configurations for pairs of triangles appropriately located with respect to the conic. (Received July 19, 1954.)

\section{1t. Valdemars Punga: Note on covariant differentiation by means of measuring vectors.}

Professor Schouten in his Ricci-Kalkül derives the formulas for the covariant differential of co- and contravariant vectors using measuring vectors $e_{(\lambda)}^{\kappa}$ and $e_{\lambda}^{(\kappa)}$, but in deriving the formula for the covariant differential of affinors of valence higher than 1 he uses ideal vectors. In this paper it is shown how the process of covariant differentiation of affinors of any valence can be developed by means of the measuring vectors. Similarly to $v^{\kappa}=v^{(\lambda)} e_{(\lambda)}^{\kappa}, w_{\lambda}=w_{(\kappa)} e_{\lambda}^{(\kappa)}, v^{(\kappa)}=v^{\lambda} e_{\lambda}^{(\kappa)}, w_{(\lambda)}=w_{\kappa} e_{(\lambda)}^{\kappa}$, the author writes for affinors of valence higher than 1 (taking as example affin $r v_{\nu}^{k \lambda}$ ): $v^{\kappa \lambda}$ $=v^{(\rho)(\sigma)}\left({ }_{(\tau)} e_{(\rho)}^{\alpha}\right)_{(\sigma)}^{\lambda} e_{\nu}^{(\tau)}, v_{(\nu)}^{(\alpha)}{ }_{(\nu)}^{(\lambda)}=v_{\tau}^{\rho \sigma} e_{\rho}^{(\kappa)} e_{\sigma}^{(\lambda)} e_{(\nu)}^{\tau}$ and for example $v_{(\nu)}^{(\kappa) \lambda}=v_{\tau}^{\rho(\sigma)} e_{\rho}^{(\kappa)} e_{(\sigma)}^{\lambda} e_{(\nu)}$. Assume that $\nabla_{\mu} e_{(\lambda)}^{\kappa}=\Gamma_{\mu(\lambda)}^{\kappa}, \delta e_{(\lambda)}^{\kappa}=\Gamma_{\mu(\lambda)}^{\kappa} d \xi^{\mu}$ and $\nabla_{\mu} e_{\lambda}^{(\kappa)}=-\Gamma_{\mu \lambda}^{(\kappa)}, \delta e_{\lambda}^{(\kappa)}=-\Gamma_{\mu \lambda}^{(\kappa)} d \xi^{\mu}$. Since $e_{(\sigma)}^{\kappa}$ and $e_{\sigma}^{(\kappa)}$ act as index-substitution operators, define $\Gamma_{\mu \lambda}^{\kappa}=\Gamma_{\mu(\sigma)}^{\kappa} e_{\lambda}^{(\sigma)}, \Gamma_{\mu \lambda}^{\kappa}=\Gamma_{\mu \lambda}^{(\sigma)} e_{(\sigma)}^{\kappa}$. Using the introduced formalism, the author derives the formulas for the covariant differentiation for affinors of any valence, affinor-densities, etc. (Received July 12,1954.) 


\section{2t. Andrew Sobczyk: Families of planes.}

The family of all lines in $n$-dimensional vector space $E_{n}$ is equivalent to the fiber bundle of the tangent hyperplanes to the sphere $S_{n-1}$. In $E_{4}$, there exist three types of covering families of planes, $U, V, W$. If $P_{1}, P_{2}$ are any two planes of a family $U$, then either $P_{1}$ and $P_{2}$ intersect in a line, or there is a third plane $Q$ of $U$ which intersects both $P_{1}$ and $P_{2}$, in lines parallel to a fixed direction. The fibering of the sphere $S_{3}$ with $S_{2}$ as base determines a family $V$ of planes, all passing through the origin and having no other common points. If $P_{1}, P_{2}$ are any two planes of a family $W$, then $P_{1}$ and $P_{2}$ have no common point (they lie in parallel hyperplanes). By means of considerations involving fiber bundles, projective duality, hyperplanes at infinity, and results of the author on families of lines, these and numerous further results are established concerning covering, intersectional, and directional properties of families of planes, especially in $E_{3}$ and in $E_{4}$. (Received July 15, 1954.)

\section{3t. Andrew Sobczyk: Simple families of lines.}

Let "non-horizontal" lines in $(n+1)$-dimensional real vector space $E_{n+1}$ be coordinatized by their intersections $x, y$ with parallel hyperplanes $H, K$. Then the equation for any pencil of lines with center $\left(w ; w_{n+1}\right)$ is $(y-w)=c I(x-w)$, where $I$ is the identity, and $c=\left(b-w_{n+1}\right) /\left(a-w_{n+1}\right), z_{n+1}=a, z_{n+1}=b$ being respectively the equations of $H, K$. In case $(n+1)$ is odd, the family $(y-w)=T(x-w)$, where $T$ is any linear transformation having only complex eigenvalues, has the property that it contains exactly one line in every "non-horizontal" direction, and that each point $\left(z ; z_{n+1}\right)$ of $E_{n+1}$ is covered by exactly one line of the family. In general if $T$ has real eigenvalues, if any, all different from 1, then any family $y=T x+u$ is included under the previous form, and the family simply covers all of $E_{n+1}$ except for one hyperplane corresponding to each distinct real eigenvalue. If one or several eigenvalues are equal to 1 , the family is a "stack" of lower dimensional families in a sheaf of parallel flats, and therefore does not contain a line in every "non-horizontal" direction. Generalization is made to families of lines in Banach spaces. (Received July 15, 1954.)

\section{LOGIC AND Foundations}

\section{4t. M. W. Al-Dhahir: The Möbius class of configurations.}

A configuration $\mathfrak{Y}\left(a_{1}, a_{2}, a_{3}\right)$ is a set of subsets $a_{1}, a_{2}, a_{3}$ in which a relation $R$ is defined. A configuration $\mathfrak{P}^{*}\left(a_{1}, a_{2}, a_{3} ; x\right)$ is a "natural extension" of $\mathfrak{A}, \mathfrak{Y} \leqq \mathfrak{Y} *$, if each element of $x$ is determined by $R$ among some elements of $\mathfrak{A}$. Two configurations $\mathfrak{A}$ and $\mathfrak{B}$ are "geometrically equivalent," $\mathfrak{A} \sim \mathfrak{B}$, if $\exists$ a configuration $\mathfrak{C} \ni \mathfrak{U} \leqq \mathfrak{E} \& \mathfrak{B} \leqq \mathfrak{E}$. Some theorems are proved; in particular $\sim$ is an equivalence relation. Then these notions are applied to configurations corresponding to some configurational propositions equivalent to Möbius theorem. It is shown that these configurations are $\sim$ to the Möbius configuration; and hence they are all "unified" into one class-the Möbius class - of configurations "leveling" with the Möbius class of propositions. Commutativity of multiplication may be characterized by any member of the class. (Received June 3, 1954.)

\section{5t. A. R. Schweitzer: Outline of a form of general analysis. Pre- liminary report.}

The content of E. H. Moore's form of general analysis (New Haven, 1910) is classified first with regard to concepts relevant to Peano-Russell logic and Boole- 
Grassman-Schroeder logic such as property, relation, class, operation, relation between classes, composition; and second with regard to mathematical subjects such as set, semi-group, number system, function, dominance of function, convergence, continuity. Moore's analysis is interpreted as the theory of systems of classes of functions based on some or all of the categories symbolized by $A L C D K_{1} K_{2} \Delta$. The first four categories are associated with convergence; the last six, with continuity. The author's outline proceeds as follows. I. Concerning classes (loc. cit., pp. 55-76). II. Concerning convergence (pp. 13-55; 76-88). III. Concerning continuity (pp. 89149). IV. Moore's "relative uniformity" of convergence is held to be a key to the unification of certain theories (pp. 13-14) expressed by a formal principle (p. 1). In connection with this principle, following Moore the author traces the genesis of Moore's memoir and its relation to integral equation theory (p. 2, note). Reference is made to the author's article in Rev. de Mét. et de Mor., Paris, 1914. (Received June 22, 1954.)

756t. A. C. Sugar: Axiomatic foundation of thermodynamics. Preliminary report.

As a first task the author considers a set of axioms for the thermodynamics of the reversible phenomena of the so-called perfect, ideal or polytropic gases. By a differentiable reversible polytropic intensive system of thermodynamics, briefly called a hyperclassical system of thermodynamics, is meant an ordered sextuple, $\Sigma=\langle S, T, q$, $\left.t_{1}, c_{v}, R\right\rangle$, which satisfies axioms A1-A5. Axiom A1. $T$ is a closed interval of real numbers. A2. $t_{1}$ is in $T$. A3. $R$ and $c_{v}$ are positive real numbers. A4. $S$ is the set of ordered pairs, $(\langle p, v\rangle)$, of all real differentiable functions (whose domains are $T$ and whose values are positive real numbers) such that for each $\langle p, v\rangle$ and every $t$ in $T$, the Riemann integral $\int_{t 1}^{t} p D v$ exists. A5. $q$ is a differentiable function, whose domain of definition is $S \times T$ (cartesian product) and whose value is a real number, such that for every $\langle p, v\rangle$ in $S$ and every $t$ in $T, q(\langle p, v\rangle, t)-\int_{t_{1}}^{t} p D v=\left(c_{v} / R\right)\left[p(t) v(t)-p\left(t_{1}\right) v\left(t_{1}\right)\right]$. The second law is a consequence of axioms A1-A5. The intriguing aspect of this axiomatization is that these axioms are really all definitions. Consequently one is confronted with the surprising result that the operation of clarification has established that this portion of thermodynamics is a science of no assumptions only definitions. Temperature is defined using the equation of state $\theta=p v / R$. Reversibility is taken as a continuity condition on the set of admissible state paths, $S . T$ is a set of real numbers measuring elapsed periods of time and $q(t)$ the amount of heat supplied to the "perfect gas" in $t$ units of time. It is planned to include isolated and more complicated systems, to axiomatize statistical thermodynamics and to reduce, in a strictly logical fashion, classical to statistical thermodynamics. (Received June 28, 1954.)

\section{Statistics and Probability}

\section{7t. A. G. Anderson: The prediction of quantitative characteristics in polygenic systems. II.}

A method devised by the author (Bull. Amer. Math. Soc. Abstract 59-3-315) is employed in a specific example for the purpose of predicting yield in hybrid corn crosses. The resulting analysis shows that the improvement over present methods of prediction, while not statistically significant, is comparable to that made by present methods over chance. (Received July 19, 1954.)

758t. G. E. Baxter: An analogue of the law of the iterated logarithm. 
Let $\left\{X_{n k}\right\}(k=1,2, \cdots, n ; n=1,2, \cdots)$ be a sequence of sequences of Bernoulli variables, mutually independent within each row, with $P\left\{X_{n k}=1\right\}=\lambda / n$ and $P\left\{X_{n k}\right.$ $=0\}=1-\lambda / n(k=1,2, \cdots, n)$. The classical Poisson theorem states that the distributions of the row sums $S_{n}=X_{n 1}+X_{n 2}+\cdots+X_{n n}$ converge to the Poisson distribution with parameter $\lambda$. It is now shown that $P\left\{\lim \sup _{n \rightarrow \infty}(\log \log n / \log n) S_{n} \leqq 1\right\}$ $=1$. Under the additional hypothesis that the family $\left\{X_{n k}\right\}$ consists entirely of mutually independent Bernoulli variables it is shown that $P\left\{\limsup \operatorname{sum}_{n \rightarrow \infty}(\log \log n / \log n) S_{n}\right.$ $=1\}=1$ and that for any positive normalizing factor $f(n), P\left\{\lim _{\inf _{n \rightarrow \infty}} S_{n} / f(n)=0\right\}=1$. (Received May 24, 1954.)

\section{9t. R. K. Getoor: On stochastic processes. I.}

Let $X(t), 0 \leqq t<\infty$, be a stochastic process with finite variance and let $L(X)$ be the smallest closed linear manifold of random variables containing all $X(t)$. It is well known that $L(X)$ is a Hilbert space. Necessary and sufficient conditions in terms of the covariance function $R(t, s)$ of $X(t)$ are given that the semi-group of translation operators $V_{t}: X(s) \rightarrow X(t+s)$ be well defined on a dense subset of $L(X)$. These conditions are then strengthened to insure that each $V_{s}$ is bounded. If $R(t, s)$ admits a representation in the form $R(t, s)=\int_{-\infty}^{\infty} \cdots \int_{-\infty}^{\infty} f\left(t ; \lambda_{1}, \cdots, \lambda_{n}\right) f\left(s ; \lambda_{1}, \cdots, \lambda_{n}\right)$ $d \alpha\left(\lambda_{1}, \cdots, \lambda_{n}\right)$, where $\alpha$ is a bounded measure on $E^{n}$, it is shown that there exist $n$ self-adjoint operators $A_{1}, \cdots, A_{n}$ densely defined, but not necessarily bounded, on an appropriate extension of $L(X)$ such that $X(t)=f\left(t ; A_{1}, \cdots, A_{n}\right) X(0)$. This representation is closely related to a result of Karhunen. (Received July 6, 1954.)

\section{0t. R. K. Getoor: On stochastic processes. II.}

Using the notation of the preceding abstract, stochastic processes whose translation semi-group $\left\{V_{s}\right\}$ consists of either symmetric or normal operators are investigated. A necessary and sufficient condition that $\left\{V_{s}\right\}$ consist of symmetric operators is that $R(t, s)=r(t+s)$ and thus are the processes of "convex exponential character" of Loève (Sur les fonctions aléatoires $d u$ seconde ordre, Note in P. Lèvy, Processus stochastiques et mouvement Brownien, Paris, 1948). For both symmetric and normal operators first the unbounded and then the bounded case is investigated. Under certain mild restrictions various integral representations and mean ergodic theorems are obtained for both these classes of stochastic processes. The relationship between these processes and stationary processes is also considered. (Received July 6, 1954.)

761t. H. T. McAdams: Factorial experiments as determinants. Preliminary report.

A relation is established between the theory of higher dimensional determinants and analysis of variance in a factorial experiment. Let $L$ be a set of $p$ elements $i, j, \cdots, m, n$ and let $A=\left(a_{i j} \ldots m n\right)$ be a $p$-dimensional matrix of order 2 in which $a_{i j \cdots m 1}=1$ and $a_{i j \cdots m 2}$ are observations from a $2^{p-1}$ factorial experiment. Consider all subsets $S_{r}\left(r=1,2, \cdots, 2^{p-1}\right)$ of $L$ containing an even number of elements, and let $D_{r}$ denote the $p$-dimensional determinant of $A$ with the elements of $S_{r}$ as the signant indices (R. Oldenburger, Higher dimensional determinants, Amer. Math. Monthly vol. 47 (1940) pp. 25-33). If $S_{\dot{r}}$ is that subset of $S_{r}$ which contains all the elements of $S_{r}$ except $n$, then $(1 / 2)^{p-1} D_{r}^{2}$ is the mean square obtained (in the usual analysis of variance) for the component identified by the elements of $S_{\dot{r}}$. The $D_{r}$ determine the coefficients for expressing the experimental data as a linear combination of orthogonal polynomials constructed from the elements of $S_{\dot{r}}$ by considering these elements as continuous variables in the interval -1 to 1 , and $(1 / 2)^{p-1} D_{r}^{2}$ is a measure of residual variance 
accumulated when the $r$ th term is rejected by a statistical test of significance. More general applications of higher dimensional determinants to experiment designs is anticipated. (Received July 19, 1954.)

762. Brockway McMillan: Information rates and the information lattice. Preliminary report.

Let $P$ be a given probability measure on a Borel field (BF) $I$. All BF below will be subfields of $I$. Using $P$, this paper defines a non-negative numerical function $H\{A$; $B \mid C\}$, where $A, B, C$ are BF. Theorem (i): Let $X_{n}\left(X_{\infty}\right)$ be the smallest BF with respect to which the variables $\left\{x_{t}, t \leqq n\right\}$ (all the variables) of a stationary stochastic process $\left\{x_{t}, t=0, \pm 1, \pm 2, \cdots\right\}$ are measurable, then $H\left\{I ; X_{n} \mid X_{n-1}\right\}$ is C. E. Shannon's $H(x)$, the entropy per symbol for this process (Bell System Technical Journal vol. 27 (1948)). Theorem (ii): Define $Y_{n}, Y_{\infty}$ similarly for $\left\{y_{t}, t=0, \pm 1, \ldots\right\}$; if all $X_{n}$ and $Y_{n}$ are closed by a simple closure operation, $H\left\{Y_{\infty} ; X_{n} \mid X_{n-1}\right\}$ $=H\left\{X_{\infty} ; Y_{n} \mid Y_{n-1}\right\}=H\left\{I ; X_{n} \cap Y_{n} \mid X_{n-1} \cap Y_{n-1}\right\}$ and the common value is Shannon's $H(x)+H(y)-H(x, y)$ (loc. cit.). This last permits defining a metric lattice whose elements are certain monotone sequences of closed BF (cf. C. E. Shannon, Proceedings of the International Congress of Mathematicians, 1950, Vol. II, p. 262). From its modularity and the formula (ii) follow several theorems of information theory. (Received July 16, 1954.)

\section{F. L. Spitzer: On a class of random variables.}

Let $Z$ be a complex valued random variable, $E$ its expectation operator, and $f(z)$ an analytic function. It is noted that the mean value theorems of analysis can be represented in the form $E[f(Z)]=f[E(Z)]$. This relation is used to define regular random variables (r.r.v.'s): $Z$ is a r.r.v. if its absolute moments exist and the defining relation holds for every polynomial $f$. It is shown that there exists a class of r.r.v.'s with independent real and imaginary parts which may, but do not have to be normally distributed. The generalization of the defining relation is $E[u(X)]=u[E(X)]$, where $\boldsymbol{X}$ is an $n$-dimensional vector random variable $X=\left\{X_{1}, \cdots, X_{n}\right\}, E(X)=\left\{E\left(X_{1}\right)\right.$, $\left.\cdots, E\left(X_{n}\right)\right\}$. It is to hold for all harmonic polynomials $u\left(x_{1}, \cdots, x_{n}\right)$, and the components $X_{i}$ are to be mutually independent and all their moments are to exist. If $n \geqq 3$ it is shown that the $X_{i}$ have to be normal random variables. (Received July 12,1954 .)

\section{TOPOLOGY}

\section{F. W. Anderson: A lattice characterization of completely regular C-spaces.}

A topological space is called a $C$-space in case each of its points is a $G_{\delta}$. Spaces of this type were first mentioned by E. W. Chittenden [Trans. Amer. Math. Soc. vol. 31 (1929)] and later were studied by C. W. Burrill [Doctoral Thesis, State University of Iowa,1951]. It is shown that the lattice of all real-valued continuous functions on a completely regular $C$-space characterizes the space. The proof employs techniques similar to those used by Shirota [Osaka J. Math. vol. 4 (1952)]. It is easily seen that locally compact Hausdorff $C$-spaces satisfy the first axiom of countability, but the converse is clearly false. An example is given of a paracompact $C$-space in which the first axiom of countability fails. Finally, it is noted that the space $T_{\Omega}$ [Hewitt, Trans. Amer. Math. Soc. vol. 64 (1948)] provides an example of a completely regular $C$ space which is not a $Q$-space in the sense of Hewitt. (Received May 17, 1954.) 
765. R. D. Anderson: Upper semi-continuous collections of arcs in one-dimensional continuous curves.

Let $M$ be a one-dimensional compact metric locally connected continuum (regarded as space) with the property that no point of $M$ separates an open subset of $M$. Let $\epsilon$ be any positive number. Then there exists an upper semi-continuous collection $G$ of mutually exclusive arcs filling up $M$ such that $G$ with respect to its elements as points satisfies the above conditions for $M$ and such that for each point $P$ of $M$ and element $g$ of $G, \rho(g, P)<\epsilon$. If, furthermore, $M$ is assumed to satisfy a special, rather strong, almost-homogeneity property, then it can be required additionally that $G$ be homeomorphic to $M$. The universal curve and a certain subset of the plane, among other sets, satisfy this homogeneity property. The demonstration of the existence of the collection $G$ is accomplished by a construction and argument in the general spirit of that used by the author in his paper Monotone interior mappings of manifolds to appear in Trans. Amer. Math. Soc. (Received July 19, 1954.)

\section{R. H. Bing: Another homogeneous plane continuum.}

An example is given of a homogeneous bounded plane continuum which is neither a point, a simple closed curve, nor a pseudo arc. Like a circle, it separates the plane and is decomposable. It may be called a circle of pseudo arcs since it has a continuous decomposition such that the decomposition space is a circle and the elements of the decomposition are pseudo arcs. It is circular in that for each positive member $\epsilon$, it is covered by a circular $\epsilon$-chain. This precisely prescribes the continuum in that if $M_{1}$, $M_{2}$ are any two circular continua such that there is a monotone interior map of each onto a circle such that the inverses of points are pseudo arcs, $M_{1}$ and $M_{2}$ are homeomorphic and each is homogeneous. (Received July 19, 1954.)

\section{Eldon Dyer: Certain transformations which reduce dimension.}

In this paper it is shown that if $G$ is a continuous collection of mutually exclusive arcs which is homeomorphic to a closed $n$-cell with respect to its elements as points, the dimension of the union of the elements of $G$ is $n+1$. It is also shown that if $G$ is a continuous collection of mutually exclusive $(n-1)$-dimensional continuous curves in the $n$-sphere $S^{n}$, then $G$ with respect to its elements as points does not contain any compact two-dimensional subspace. With the aid of the first of these theorems, it is shown that there does not exist a continuous collection of mutually exclusive arcs filling up either $S^{3}$ or $E^{3}$. (Received July 19, 1954.)

\section{J. B. Giever: Cohomology groups for a ring of continuous func- tions.}

Let $X$ be a locally compact Hausdorff space, $R$ the Banach algebra of real-valued continuous functions on $X$ which vanish at infinity, and $R_{*}$ the set of nonzero elements of $R$. Define $\Phi^{n}(R, G)=\left[\phi \mid \phi: R_{*}^{n+1} \rightarrow G\right]$ and define the coboundary $\delta \phi \in \Phi^{n+1}$ as usual. The function $\phi\left(r_{0}, \cdots, r_{n}\right)\left(r_{i} \in R_{*}\right)$ has empty support if and only if there is a family $\mathfrak{A}=\left\{I_{\alpha}\right\}$ of closed ideals which generate $R$ such that $\phi\left(r_{0}, \cdots, r_{n}\right)=0$ whenever $r_{i} \in I_{\alpha} \in \mathfrak{Q}$. Denoting the group of functions with empty support by $\Phi_{0}^{n}$, the group of cochains is $\Phi^{n} / \Phi_{0}^{n}$ and cohomology groups are defined as usual. $H^{n}(X, G) \approx H^{n}(R, G)$ as follows. The correspondence between open sets, $O \subset X$, and the closed ideals of functions which are zero on the complement of $O$ can be used to build a Cech type cohomology theory on $R$. Dowker's paper on the Homology groups of relations (Ann. of Math. 1952) provides the transition to the above defined Alexander type coho- 
mology groups. The above groups serve to supplement the homology groups defined by Hu (Bull. Calcutta Math. Soc. 1950). (Received July 13, 1954.)

\section{S. T. Hu: On fiber spaces with cross-sections.}

Let $p . E \rightarrow B$ be a fiber space in the sense of Serre and assume that there is a crosssection $q: B \rightarrow E$. Then $B$ can be identified with the subset $q(B)$ of $E$ and $q$ becomes the inclusion map. Let $b_{0}$ be a given point in $B$ and $F$ the fiber $p^{-1}\left(b_{0}\right)$. Then it is well known that $\pi_{n}\left(E, b_{0}\right) \approx \pi_{n}\left(B, b_{0}\right) \times \pi_{n}\left(F, b_{0}\right)$. The main purpose of this paper is to generalize this formula for the relative homotopy groups. Let $A \subset B$ and $D=p^{-1}(A)$. Then $B \cap D=A$ and, for each $n \geqq 2, \pi_{n}(E, A) \approx \pi_{n}(B, A) \times \pi_{n}(D, A) \approx \pi_{n}(B, A)$ $\times \pi_{n}\left(F, b_{0}\right)$. This is an easy consequence of the following exact sequence proved in this paper $\cdots \rightarrow \pi_{n+1}\left(E, A_{0}\right) \rightarrow^{\Delta} \pi_{n}\left(A, A_{0}\right) \rightarrow^{I *} \pi_{n}\left(B, A_{0}\right) \times \pi_{n}\left(D, A_{0}\right) \rightarrow^{J *} \pi_{n}\left(E, A_{0}\right)$ $\rightarrow \cdots \rightarrow \pi_{2}\left(A, A_{0}\right) \rightarrow^{I *} \pi_{2}\left(B, A_{0}\right) \times \pi_{2}\left(D, A_{0}\right) \rightarrow J * \pi_{2}\left(E, A_{0}\right)$, where $A_{0}$ is any subset of $A$. Further, if $\pi_{1}\left(A, A_{0}\right)=0$, then $J^{*}$ maps $\pi_{2}\left(B, A_{0}\right) \times \pi_{2}\left(D, A_{0}\right)$ onto $\pi_{2}\left(E, A_{0}\right)$. Here $\pi_{1}\left(A, A_{0}\right)$ is not a group but a set of homotopy classes of paths defined in an obvious way. (Received July 12, 1954.)

\section{F. B. Jones: On a certain type of homogeneous plane continuum.}

An example is given to show the existence of a class of compact, homogeneous plane continua each of which is decomposable and separates the plane but is not a simple closed curve. Some of the properties of these continua are developed. For example, each continuum in this class may be decomposed by an upper semicontinuous collection (of continua) into a simple closed curve. (Received July 19, 1954.)

771t. Jean-Pierre Meyer: Classification of mappings of a 3-complex into the projective plane.

This paper is concerned with the homotopy classification of mappings of a 3-dimensional complex into a 2-dimensional projective space. The method is patterned after that of Steenrod, and uses the obstruction theory with local coefficients (Olum, Ann. of Math. vol. 52). Certain functional operations, introduced by Massey (Bull. Amer. Math. Soc. vol. 55, p. 303), and variants thereof, are also used. If $(K, L)$ is a simplicial pair with $\operatorname{dim} K \leqq 3$ and $H^{3}(K, L ; Z)$ has no element of order 2, then the mappings $K \rightarrow P^{2}$ which agree on $L$ are completely classified. If $(K, L)$ satisfies a further condition, effectively computable invariants are defined which enable us to determine whether two such mappings are homotopic rel $L$. As an application of the above, a complete and effective classification of mappings of compact orientable 3manifolds with finite Abelian fundamental groups into 2-dimensional projective space is obtained. (Received April 22, 1954.)

\section{2t. J. T. Mohat: Concerning spirals in the plane.}

It is shown that if, in the plane, $M$ is an inner limiting set such that (1) for every point $P$ and positive number $\epsilon$ there exists a simple closed curve of diameter less than $\epsilon$ enclosing $P$ and containing no point of $M$ and (2) there is a line $l$ such that the projection of $M$ onto $l$ is a connected open subset of $l$ and no perpendicular to $l$ contains two points of $M$, then if $A B C D$ is a rectangle there exist an equicontinuous collection $G$ of mutually exclusive arcs and a point set $K$ such that (1) each arc of $G$ has one end point in the segment $B C$ and the other in the segment $A D$, lies, except for its end points, in the interior of $A B C D$, and spirals down on only one point, (2) every point of the interior of $A B C D$ belongs to some arc of $G$, (3) a point belongs to $K$ if, 
and only if, some arc of $G$ spirals down on it, and (4) $M$ is topologically equivalent to $K$. (Received August 10, 1954.)

\section{P. S. Mostert: On locally connected homogeneous spaces.}

Theorem. Let $X$ be a locally connected, locally compact Hausdorff space of finite dimension which admits a locally compact, transitive transformation group satisfying the second axiom of countability. Then $X$ is homeomorphic to an analytic manifold. CoroLLARY. Let $X$ be a locally compact, locally connected, connected Hausdorff space of finite dimension. Then $X$ is homeomorphic with a metrically homogeneous space if and only if it is homeomorphic with the space of cosets of a Lie group modulo a compact subgroup. The proof of the theorem involves, principally, the use of a generalization of the author's local cross section theorem for locally compact groups (Proc. Amer. Math. Soc. vol. 4 (1953) pp. 645-649). (Received July 15, 1954.)

774. H. M. Trent: $A$ note on the enumeration and listing of all possible trees in a connected linear graph.

This paper presents theorems which provide efficient means for determining the number of distinct trees in any connected linear graph and for the specification of each of these trees. In each case the results depend only on the expansion of a single determinant. Dual theorems are presented for the enumeration and listing of all complements to trees. These theorems were discovered independently by Okada and Onodera but were published in a not readily available source (Bull. Yamagata University, Natural Science, Vol. 2, No. 2). The proofs of the theorems have been materially shortened. (Received July 15, 1954.)

\section{P. M. Swingle: $n$-indecomposable and related connexes.}

A set $W$ is an essential sum of the elements of a class $(C)$ of subconnexes if $\mathrm{U}(C)$ $=W$ and no $C$ of $(C)$ is contained in the closure of the sum of the rest. A connexe $W$ is $n$-indecomposable if it is the essential sum of a class of $n$, but not more than $n$, subconnexes. For an integer $n>0$, the compact connexe $W$ is $n$-indecomposable if and only if it is the essential sum of $n$ indecomposable subconnexes and is an irreducible joining connexe closure about a finite set. (See C. E. Burgess, Proc. Amer. Math. Soc. vol. 4 (1953) Theorem 5, p. 237; for definitions see P. M. Swingle, Bull. Amer. Math. Soc. vol. 47 (1941) p. 797). If the compact connexe $W$ is $n$-indecomposable, $n$ as above, $x \in$ (closure of $W$ ), then there exist integers $n_{x} \leqq n$ and $2 \leqq m_{x} \leqq n$ such that $W$ is locally $n_{x}$-indecomposable at $x$ and locally $m_{x}$-irreducible at $x$; also then the set points where $W$ is locally 1 -indecomposable are dense in $W$. If $W$ is a finitely-indecomposable connexe in a completely separable space then there exists a set $P$ of power aleph-zero such that $W$ is an irreducible joining connexe closure about $P$. (Received March 11, 1954.)

\section{W. R. Utz: Equicontinuity and the limits of orbits.}

Pointwise periodicity, pointwise almost periodicity, and pointwise recurrence under equicontinuous and equi-uniformly continuous homeomorphisms of a metric space onto itself are studied to compare an equicontinuity hypothesis on the homeomorphism with the hypothesis that the metric space be compact. These two hypotheses are compared in several theorems on extensions from sets dense in the metric space to the entire space and in theorems concerning the limits of sequences of orbits under a given homeomorphism. For example, if $X$ is a metric space, $T(X)=X$ is an 
equicontinuous homeomorphism and if $\alpha_{i}$ is a sequence of point orbits under $T$ such that $\lim \alpha_{i}=\beta$, then $\beta$ is the closure of the orbit of each of its points and hence is a minimal set. From this it follows that if $T$ is pointwise periodic, then $\beta$ is a (periodic) point orbit. This is similar to part of a theorem due to G. E. Schweigert [A note on the limit of orbits, Bull. Amer. Math. Soc. vol. 46 (1940) pp. 963-969; cf. also D. W. Hall and G. E. Schweigert, Properties of invariant sets under pointwise periodic homeomorphisms, Duke Math. J. vol. 4 (1938) pp. 719-724, and G. T. Whyburn, Analytic topology, New York, 1942, p. 259] in which $X$ is assumed compact but $T$ is only assumed to be a homeomorphism. The remainder of Schweigert's theorem (pertaining to component orbits) does not hold if compactness of $X$ is replaced by equicontinuity of $T$. (Received July 15, 1954.)

\author{
W. T. Puckett, \\ Acting Associate Secretary
}

